\title{
Les fondations du royaume de 'Uvea
}

Une histoire à revisiter

\section{Bernard Vienne et Daniel Frimigacci}

\section{(2) OpenEdition}

Journals

Édition électronique

URL : http://journals.openedition.org/jso/529

DOI : $10.4000 /$ jso. 529

ISSN : $1760-7256$

Éditeur

Société des océanistes

Édition imprimée

Date de publication : 1 décembre 2006

Pagination : 27-60

ISSN : 0300-953x

Référence électronique

Bernard Vienne et Daniel Frimigacci, «Les fondations du royaume de 'Uvea », Journal de la Société des Océanistes [En ligne], 122-123 | Année 2006, mis en ligne le 01 décembre 2009, consulté le 01 mai 2019. URL : http://journals.openedition.org/jso/529; DOI : 10.4000/jso.529 


\title{
Les fondations du royaume de 'Uvea. Une histoire à revisiter ${ }^{1}$
}

par

\author{
Bernard VIENNE* et Daniel FRIMIGACCI**
}

\begin{abstract}
RÉSUMÉ
Dans la perspective d'une anthropologie historique qui se construit sur un questionnement de l'histoire, les données de l'archéologie et de l'ethnohistoire de 'Uvea sont ici présentées en se référant à une périodisation qui retient quatre phases et une chronologie recalées dans celle régionale de la Polynésie occidentale. Cette mise en perspective diachronique conduit à s'interroger sur la nature et les déterminants du processus d'évolution culturelle et de transformation sociale qui aboutit, à partir des découvreurs Lapita à la formation du "royaume " polynésien de 'Uvea. Dans cette "histoire du long terme », cette dynamique historique d'adaptation, place est aussi faite aux stratégies des " acteurs de l'histoire 》 et au rôle joué par "l'événement».
\end{abstract}

Mots-CLÉS : ethnoarcheologie, Polynésie occidentale, Lapita, chefferies, occupations, forts, sépultures.

\begin{abstract}
In the so called historical anthropological perspective which involves a questioning of the historical data itself, the archeological findings and the ethnohistorical records from 'Uvea island are reevaluated according to a four stages sequence previously established for 'Uvea and a chronology embedded in a regional sequence with reference to Tonga and Futuna. The nature of the dynamic process of evolution and cultural differentiation from the earlier Lapita settlements to the late centralized proto-kingdom of 'Uvea is evaluated in a way which outlines the importance to be conceded to the "events» and the "actors' strategies» of history.
\end{abstract}

KeYwords: historical anthropology, Western Polynesia, Lapita, chiefdoms, fields monuments, burials.

1. Faute d'un accord sur une terminologie plus appropriée, nous employons le terme de « royaume », par opposition à celui de « chefferie », pour distinguer une organisation territoriale politiquement intégrée sous l'autorité (réelle ou idéologique) d'un pouvoir central, avec ou non délégation institutionnelle du pouvoir exécutif à une autorité administrative constituée d'une organisation territorialement et politiquement segmentée du politique, couramment connotée par le terme de "chefferie » ou " chefferie à titres ». Cette distinction ne préjuge pas de la structure «segmentaire » ou " ramifiée » de groupes de parenté résidentiels que l'on peut observer dans l'un ou l'autre cas et qui en constituent le tissu social, ni de la structure hiérarchique des statuts ( $c f$. Sahlins, 1958). On se reportera à Daniel Frimigacci et al. (1982 et 1984) et Daniel Frimigacci (2000) pour l'inventaire des sites répertoriés, leur localisation précise et la présentation du matériel archéologique. La référence au Corpus de traditions orales renvoie aux traditions recueillies et enregistrées lors des missions ORSTOM-CNRS (1982/1984) se rapportant à l'inventaire des sites archéologiques et ethnohistoriques de l'île de 'Uvea (Frimigacci et al., 1982 et 1884).

* Ethnologue à l'IRD-Nouméa, UR 092 ADENTRHO (Adaptations humaines aux environnements tropicaux durant l'Holocène), vienne@lagoon.nc

** Archéologue à l'IRD-Nouméa, UR 092 ADENTRHO, dfrimigacci@canl.nc 
Hau ta kumi kau tala Kau talu atu Fatuloamaka Fatuloamaka mo Fuilaoa Na tu'u i te ala ki' Ahoa Kua tuku maka o fakailoga Ko te kumi o tana talanoa Ko te Tu'i o 'Uvea katoa. ${ }^{2}$

Comme le laisse entendre le titre, l'objet de cet article n'est pas de présenter le résultat de nouvelles recherches empiriques sur la préhistoire et l'ethnohistoire de 'Uvea, mais plutôt d'approfondir une réflexion commune, initiée dès les années 1980, lorsque ont débuté nos recherches sur le terrain. Les données que nous avons recueillies et les acquis de nos travaux résultent d'une tentative d'intégrer dans une même problématique de mise en perspective historique les questionnements, les méthodes et les apports de l'archéologie et de l'anthropologie. Nous avons choisi ici un positionnement critique qui se nourrit des nouveaux questionnements que suggère le résultat de recherches plus récentes tant dans nos domaines que dans des domaines connexes. D'une part, comment doit-on revoir nos données empiriques et réviser nos analyses à la lumière des apports nouveaux de la recherche? D'autre part, qu'est-ce que le dossier 'Uvea peut apporter au développement de ces nouvelles problématiques? Dans quelle mesure nos données confirment ou infirment certaines hypothèses nouvelles sur le peuplement, la différentiation culturelle, la transformation des sociétés insulaires du Pacifique occidental ? À l'inverse, les nouveaux acquis de la recherche océanienne doivent-ils nous pousser à reconsidérer aujourd'hui certaines hypothèses, à rejeter ou nuancer certaines interprétations et selon quelles perspectives? Enfin, si l'on veut bien adhérer à l'idée que «l'histoire est le mouvement par lequel une société se révèle pour ce qu'elle est » (Dumont, 1957: 21, notre traduction), on comprendra mieux le sens de notre démarche.

\section{Les données contextuelles de l'histoire de 'Uvea}

\section{Anthropisation des milieux naturels}

L'évolution des milieux naturels sous l'action de l'homme constitue une diachronie sur le "long terme», une histoire des «rythmes lents », qui peut nous renseigner sur l'évolution des modes de pensée, d'organisation et de gestion des milieux naturels et des biotopes, et par là même s'avérer un indicateur des transformations sociales et des dynamiques démographiques. Elle permet aussi de mieux apprécier le poids des contraintes induites par l'insularité dans ce contexte culturel. Bien que de tels questionnements aient été déjà au centre des préoccupations de Patrick Kirch (1976a, 1976b), il reste encore beaucoup à faire dans ce domaine de la recherche, aussi nous n'évoquerons que les aspects qu'il nous semble pertinent de prendre en compte.

Les équilibres de l'écosystème de 'Uvea à cette période de l'Holocène tels qu'on peut s'en faire une idée, les restituer et les comprendre, ont été profondément modifiés au cours de cette brève histoire par divers facteurs dont des évolutions climatiques, sans doute des catastrophes naturelles, et, bien sûr, les impacts de l'activité humaine sur un milieu naturel réputé fragile. C'est une dimension qu'il faut prendre en compte pour une relecture de l'ethnohistoire de 'Uvea. La relation identitaire d'ordre structural (Vienne, 1998 ; Bonnemaison, 1996 ; Kirch, 1984 et 1994) que l'on peut repérer entre le changement d'organisation et de gestion des espaces insulaires et une transformation majeure de l'organisation sociale, l'émergence d'un système structuré de "chefferies", s'inscrit dans la limite des contraintes imposées par cette histoire des milieux naturels.

Cette île basse, d'origine volcanique, dont le point culminant atteint seulement $151 \mathrm{~m}$ d'altitude, est entourée d'un récif barrière comprenant quatre passes toutes situées au sud-ouest et à l'ouest. L'île est cernée d'une étroite bande de terre fertile constituée de terrains argileux ou le plus souvent d'une dune argilo-sableuse en bordure d'un ravin qui entoure parfois l'île, vestige d'un soulèvement très ancien. Le rivage marécageux, à quelques mètres en contrebas du ravin, est occupé par la mangrove, notamment des palétuviers (Bruguiera gymnorrhiza L.). Dès que l'on s'éloigne du bord de mer, on rencontre très

2. «Venez entendre notre discours / Nous parlons de la pierre Fatuloamaka / Fatuloamaka et Fuilaoa / Qui se dresse sur le chemin de 'Ahoa / Une pierre laissée là comme témoin / C'est le sens même de son histoire / C'est le chef suprême de tout 'Uvea " (Burrows, $1945:$ 57, notre traduction). 
vite un plateau fortement latérisé : le toafa. Nos relevés sur le terrain ont montré l'absence totale de vestiges d'occupation humaine permanente sur ce toafa, ce qui laisserait à penser que cet espace « désertifié », colonisé notamment par les fougères, les pandanus, les Hibiscus tiliaceus et les Casuarina equisetifolia ne serait pas d'origine anthropique. Ces observations ont été corroborées par une mission pédologique entreprise par l'IRD au cours des années 1980 (Beaudou, communication personnelle) et l'étude des formations végétales (Brisse et Hoff, 1990; Guiot, 1997). Il n'y a pas de rivières permanentes, seulement des couloirs où dévalent parfois les eaux générés par de violents orages. L'eau est donc une préoccupation. Hormis les lacs, nous avons répertorié sur la bande de terre fertile, et donc propice à l'horticulture, du bord de mer vingttrois sources, douze puits et dix-huit tarodières dont trois sont alimentées par des sources, les autres étant alimentées par la nappe phréatique ou encore par les formations marécageuses du bord de mer comme la tarodière de To'oga toto (MU102).

Préalablement à l'intensification de la culture irriguée du taro (Colocasia esculenta), essentiellement sur la côte est et nord-est de l'île, permise par les grands aménagements hydrauliques, les ressources en eau et les meilleures terres de cultures se trouvaient concentrées dans la moitié sud de l'île, l'horticulture étant ici fondée sur la prééminence de la culture de l'igname 'ufi (Dioscorea spp.), du kape (Alocasia macrrorhiza) associée au bananier (Musa spp.) et à l'arbre à pain (Artocarpus altilis) ${ }^{3}$. C'est là un contexte qui a permis de plus fortes densités démographiques et favorisé le développement de « chefferies » fortes dans le sud et non dans le nord.

Le passage d'une économie organisée autour de la pêche, de l'exploitation récifale et de la collecte (Frimigacci 1980), à une économie d'horticulteurs sédentarisés, dans un contexte de croissance démographique forte, induit un impact sans précédent sur l'environnement en particulier sur les ressources récifales et le couvert forestier ; une activité humaine qui entraîne des changements dans un environnement dont l'homme n'a plus entièrement le contrôle. Nous faisons l'hypothèse que la transition - d'ailleurs progressive - entre la période Utuleve I et Utu- leve II (voir plus avant $\S$ Chronologie et périodisation) traduit cette transformation des rapports entre l'homme et le milieu insulaire corollaire d'une mutation des implantations humaines et de l'organisation des espaces. Si on pousse un peu plus avant l'analyse, elle semble bien aussi coïncider avec la redéfinition des grands réseaux de communications trans-océaniens ${ }^{4}$.

L'ethnographie minutieuse des pratiques horticoles, les ethnosciences et la complexifications des modalités de gestion et de contrôle des environnements laissent à penser que les populations de 'Uvea ont progressivement pris conscience de ces dépendances et acquis en conséquence une certaine compréhension de la préservation des équilibres écologiques naturels qui a été formulée tant sur le plan institutionnel qu'idéologique - réglementation de l'accès aux ressources, croyances religieuses, sacralisation des espaces, etc. (Burrows 1937 ; Guiot 1997 ; Vienne 1999). On en déduit une maîtrise relative de leurs modalités d'adaptation au milieu naturel qui a pu favoriser certaines innovations technologiques (irrigation) ou institutionnelles (contrôle des moyens de production).

La maîtrise et le contrôle de l'eau, et la disponibilité devenue limitée des terres de cultures pour cause de croissance démographique, sont deux des facteurs en rapport avec l'environnement qui ont pu susciter le développement de l'irrigation et l'aménagement de grandes tarodières sur la côte est, partout où cela était possible. Cette transformation est probablement récente, contemporaine de la mise en place du système dynastique, de l'abandon de la poterie et de la généralisation d'un nouveau modèle d'occupation et d'organisation de l'espace. L'étude du foncier confirme cette corrélation entre une transformation sociale majeure - la formation du royaume de 'Uvea - et une réorganisation de l'espace et du système de production. Rappelons cependant que cette concordance entre le développement des grands systèmes d'irrigation et une organisation hiérarchisée du politique n'est en rien systématique en Océanie comme le montrent les tarodières irriguées de Vanua Lava aux îles Banks ou de la côte nord ouest de Santo, au Vanuatu, ou même certains dispositifs des îles Salomon et de la NouvelleCalédonie. Elle est à l'évidence médiatisée par d'autres déterminations.

3. Une culture sur brûlis avec cycles de jachères longues et préservation d'espaces forestiers intercalaires. Malgré l'importance prise par la culture irriguée du taro (Colocasia esculenta), cet équilibre est resté au centre du système agraire. Les cultivars hybrides d'espèces sauvages pour le bananier et certaines variétés archaïques d'arbre à pain soulignent l'ancienneté et l'importance de ces deux plantes alimentaires. Cf. Jacques Barrau (1963), Bernard Vienne (1999) et Atoloto Malau (1999, 2004).

4. Vu la complexité de cette problématique régionale, nous ne ferons que mentionner un point que nous nous réservons de développer et argumenter dans une autre publication. 
L'élevage du cochon, dont l'importance économique mais surtout sociale et cérémonielle est aujourd'hui considérable, pourrait être de pratique relativement récente, une innovation concomitante du développement des "chefferies à titres » ou de la mise en place de la « royauté ». Il n'a pas été trouvé de vestiges de cochon dans les fouilles à Wallis. L'absence constatée de barrières entre cet animal et les cultures, notamment les tarodières, laisse penser qu'il n'est peut-être pas présent sur l'île depuis la mise en place probable de l'horticulture à la fin de l'Utuleve I (fig. 6). Encore faut-il faire la différence entre les tarodières de type eau entourant la terre (type 'Uvea), moins fragiles à cet égard que celles de type terre entourant l'eau (type Futuna). Les cochons sont élevés en liberté en bord de mer sur le platier récifal ou la zone marécageuse qui borde la zone d'habitation. C'est l'organisation des api résidentiels, de l'habitat, qui protège les cultures et joue le rôle de barrière contre le vagabondage des cochons. Cependant, trois dents de cochon proviennent de la fouille d'Utuleve mais elles se trouvaient dans un environnement stratigraphique remanié (Balouet et al., 1987).

\section{'Uvea dans le Pacifique ouest: les réseaux de communication transocéaniques}

À l'exception peut-être de brèves périodes de son histoire, 'Uvea n'a pas évolué en vase clos. Elle faisait partie intégrante d'un environnement insulaire. Par contre les relations de 'Uvea avec les archipels voisins ont, elles, changé dans le temps. Les réseaux se sont confortés ou distendus. Certaines relations se sont réorientées à des périodes charnières de l'histoire de l'île. La nature de ces relations transocéaniques semble même avoir évolué en fonction de la formation et du développement de ce royaume polynésien. Nous avons acquis la conviction que ces échanges interinsulaires - dont l'étude reste encore largement à faire - ont été déterminants dans la dynamique des transformations sociales et de la différenciation culturelle. En comprendre mieux l'organisation semble alors essentiel pour retracer la configuration des diverses formations sociales qui se sont succédé à 'Uvea. L'ouverture vers l'extérieur est, au même titre semble-t-il que les transformations des modes d'occupation et d'organisation de l'espace insulaire, un marqueur dans le temps d'un processus permanent d'adaptation et d'ajustement à un environnement écologique insulaire naturellement appauvri, extrêmement fragile et précarisé par des facteurs anthropiques aussi considérables que le développement de l'horticulture sur brûlis ou la croissance démographique des populations qui s'y sont sédentarisées ${ }^{5}$. Cette ouverture permanente vers le monde extérieur pourrait être l'une des conditions requises pour le maintien d'une forte croissance démographique ${ }^{6}$ en dépit des aléas naturels. Patrick Kirch (1984) voit dans cette forte croissance démographique un facteurclé de l'émergence et de la formation des royaumes polynésiens à partir de cette « communauté ancestrale de culture " (Golson, $196: 176$ ) qui préside aux origines des sociétés de la Polynésie occidentale. Nos données confortent cette hypothèse pour 'Uvea. Le maintien, la dislocation et/ou la réorganisation dans le temps des relations interinsulaires et une certaine forme de compétition en vue de contrôler les échanges avec l'extérieur, constituent peut-être l'une des dimensions critiques de l'histoire pré-européenne de 'Uvea, tant pour fixer le contexte politique et culturel des différentes époques que pour en comprendre l'évolution. Les voyages tiennent une place importante dans la tradition orale que ce soit allusivement, symboliquement ou in texto, conférant à ceux qui les entreprennent un prestige accru du fait même de leur réussite.

On peut schématiser l'histoire de ces relations interinsulaires transocéaniques en les présentant dans le temps autour de ce qui apparaît comme trois systèmes organisés de relations suivies qui se sont succédé dans la chronologie et qui coïncident sensiblement avec des évolutions/transformations du système social lui-même ${ }^{7}$.

\section{- Le réseau des échanges interinsulaires " Lapita $»^{8}$}

Les conditions du peuplement de l'île par les «découvreurs Lapita», les colonisateurs, et les vestiges mis au jour dont témoignent le sites d'Utuleve et d'Utupoa (Frimigacci et al.,1982 et 1984 ; Frimigacci, 2000) confortent l'image d'un peuplement relativement homogène et concomitant de la formation de ce « berceau polynésien » que constituent les archipels de Fidji, Tonga,

5. Sur cet aspect des rapports entre le processus de peuplement et l'appauvrissement relatif des milieux naturels en fonction de l'éloignement des îles voir les analyses heuristiques de Matthew Spriggs (1997).

6. Une forte densité évoquée avec constance par la tradition orale.

7. Le problème est ici vu du point de vue de 'Uvea. Il en serait sans doute sensiblement différent si l'on privilégiait le point de vue tongien, samoan ou fidjien.

8. Parler de « réseaux d'échanges Lapita » peut sembler abusif; il vaudrait sans doute mieux évoquer des « circulations maritimes » pour souligner le fait que la navigation s'est effectuée selon des « itinéraires repérés » et que des objets ont circulé sur de longues distances. 
Samoa, Rotuma, 'Uvea et Futuna. On peut penser que cette sorte de « mer intérieure » qui délimite le pôle sud-ouest du grand axe de peuplement qui mena les navigateurs Lapita de l'archipel Bismark à la Polynésie occidentale via le Nord des Salomon, les Santa Cruz et le Vanuatu ait permis, selon toute vraisemblance et au moins jusqu'à la généralisation attestée de l'horticulture, le développement et le maintien de relations suivies entre ces archipels eu égard à leur relative proximité. Une rupture des relations en retour le long de l'axe mélanésien Fidji Bismark serait néanmoins intervenue assez rapidement. L'absence de verre volcanique et d'obsidienne et d'autres items indicateurs des « réseaux d'échanges Lapita» (Kirch, 1990), plaide pour une rupture - au moins temporairedes communications, une période d'autarcie plus ou moins longue consécutive de la mise en valeur horticole de l'île. C'est là une hypothèse.

La pénétration des milieux insulaires, l'occupation des espaces intérieurs, la maîtrise de nouveaux écosystèmes dus au développement rapide de cette horticulture vont diversifier les modalités d'occupation de l'espace, générer de nouveaux modèles pour en penser l'organisation et la gestion et remodeler, redessiner, les relations interinsulaires. L'argument reste certes largement hypothétique - et nous en sommes conscients - vu l'état actuel de nos connaissances. Il faudrait pouvoir mieux cerner dans chaque cas comment s'est effectuée cette transition d'une adaptation très spécifique à un écosystème essentiellement marin, similaire à toute cette région, qui caractérise les découvreurs Lapita (poterie décorée), vers une adaptation centrée sur l'horticulture (et accessoirement l'élevage), développant des modalités spécifiques d'organisation et de gestion des espaces et des écosystèmes, générant progressivement des identités différenciées sur un fond culturel commun. Les données de la reconstruction linguistique et certaines affinités culturelles laissent entendre une communication orientée, dans un premier temps, beaucoup plus vers les Samoa, Futuna, et probablement Rotuma, que vers Fidji et Tonga (Burrows 1937 ; 1938a ; 1939 ; Pawley et Green, 1984 ; Pawley et Ross 1993) ${ }^{9}$. La venue ultérieure de populations intrusives importantes en provenance des Tonga aurait assez rapidement réorienté les relations de 'Uvea avec l'extérieur, vers Niua et l'archipel des Tonga ${ }^{10}$.
- Le « berceau polynésien » : un réseau resserré de relations maritimes

Il paraît vraisemblable que le passage d'une économie centrée sur la pêche et la collecte récifale à une économie plus étroitement dépendante de l'horticulture ait eu, entre autres conséquences, un repli autarcique provisoire. À 'Uvea, il semble s'accompagner d'une croissance démographique importante - et/ou d'un apport marginal de populations extérieures - si l'on en juge par l'extension très rapide du peuplement et l'extrême densité relative des tessons de poterie (poterie non décorée de type Utuleve II et surtout Utuleve III) collectés en surface (voir plus avant $\S$ chronologie et périodisation ; Frimigacci et al., 1982 et 1984) .

La rupture des communications en retour avec Fidji et la Mélanésie le long de l'axe de communication des réseaux d'échange Lapita pourrait être le corollaire de cette pénétration des milieux insulaires et de leur mise en valeur. Elle pourrait aussi coïncider sur le plan diachronique avec une réorientation des échanges entre les Santa Cruz et le Nord Vanuatu, via les îles Banks (Green, 1976 ; Ward, 1979 ; Kirch et Yen, 1982). Il s'en suivra la mise en place d'un nouveau réseau de communications interinsulaires dans le triangle Fidji, Tonga, Samoa, incluant Futuna et sans doute Rotuma. Les communications dans cette région du « berceau polynésien » vont ensuite se formaliser, se densifier, et les sociétés de la Polynésie occidentale évoluer plus ou moins en « vase clos " et afficher chacune pour elle-même une identité propre dans une même "communauté de culture », jusqu'à l'expansion nouvelle du royaume de Tonga en direction de la Mélanésie du Sud ${ }^{11}$.

\section{- Des relations privilégiées avec Tonga}

Dés le règne du Tu'i Tonga Takalaua jusqu'à l'arrivée des Européens dans cette région du Pacifique ouest, Tonga et 'Uvea entretiendront des relations suivies. Elles s'articulent autour de trois grands thèmes :

1. L'intégration territoriale et «politique », les tentatives d'inscrire de manière plus ou moins étroite l'île de 'Uvea dans l'espace territorial tongien sous le contrôle des Tu'i Tonga.

2. L'intégration lignagère par des stratégies matrimoniales visant à réinsérer la structure seg-

9. Voir Edwin G. Burrows (1937 : 172) : «Uvean culture, then, is western Polynesian. It is mainly Tongan, but shows traces of a pre-Tongan population and of minor influences from other Polynesian islands».

10. C'est ce que pourrait laisser entendre le leitmotiv dans la tradition orale d'un originel tongien des valeurs, des façons de faire et des institutions de 'Uvea (cf. Corpus de traditions orales [cf. note 1] et Henquel [1910]) qui est loin d'être toujours évident.

11. En direction de l'île des Pins, du Sud Vanuatu, des îles Banks notamment (Guiart, 1963). 
mentaire de la société de 'Uvea dans le système ramifié tongien.

3. Des relations d'échanges et d'ordre politico-cérémoniel que symbolisent par exemple l'exportation des « dalles de corail » servant aux sépultures des Tu'i Tonga - et autres 'eikiainsi que la participation aux rites funéraires, les négociations autour du fatogia dû aux Tu'i Tonga, et les manquements aux cérémonials qu'évoque si souvent la tradition orale de 'Uvea (cf. Corpus de traditions orales et Henquel, 1910).

La tradition tongienne nous informe sur le processus de formation du "royaume maritime » des Tu'i Tonga, les innovations sous le règne de Tu'i Tonga Takalaua et les réformes institutionnelles de Kau'ulufonuafekai (Gifford, 1929 ; Bott, 1982 ; Herda, 1988 ; Kirch, 1984 ; Mahina, 1992). La dualité tu'ilhau apparaît sous le règne de Kau'ulufonuafekai, au dix-huitième niveau généalogique, suite à une segmentation et à la création du premier titre de Tu'i Ha'a Takalaua pris par le frère cadet de Kau'ulufonua dénommé Mo'ungamotu'a. Une deuxième segmentation, à l'origine du titre Tu'i Kanokupolu s'effectuera au quatorzième niveau généalogique, soit à l'époque de Takumasiva. L'instauration du hau, la collecte du fatogia et le rôle déterminant que jouent les deux grands lignages tongiens Ha'amea et Ha'avakatolo ${ }^{12}$ dans la mise en place de ces institutions concernent directement l'histoire de 'Uvea.

«The Tu'i Ha'atakalaua was known as the hau, which means "victor, conqueror" in Tongan, and has also come to mean "the one who rules, sovereign". The term was first used for the Tu'i Ha'atakalaua, then later for the Tu'i Kanokupolu. Later still, in the troubled times of 'Ulukalala's wars, Finau 'Ulukalala was known as hau in Ha'apai and Vava'u, and various other title-holders (Vaha'i, Teukava, Tâkai) were successfully known as hau in parts of Tonga. It must be understood that hau means the secular king; the sacred king was of course the Tu'i Tonga... The general duties of the Tu'i Ha'atakalaua were to guard and protect the Tu'i Tonga, to see that the 'inasi was contributed, and to maintain order in the nation. » (Bott, 1982 : 109)

C'est en raison de ce contexte qu'intervient la première tentative d'intégration territoriale de 'Uvea dans la sphère d'influence tongienne.

À l'instar d'autres îles et archipels de «l'empire» tongien, l'île de 'Uvea fut le lieu d'affrontements - domination territoriale mais surtout compétition de prestige et pour le pou- voir politique - entre au moins deux des grands lignages (ou " groupes tribaux ») tongiens, les Ha'a Lo'au et les Ha'a Falefisi, par le biais de leurs segments Ha'amea et Ha'avakatolo ${ }^{13}$.

« Traditionally it was a Loau who allotted stewards to all Tonga as far as Uvea, and who allotted the first tasks (fatongia) among the different people. » (Gifford, 1929 : 131)

Avec la mise en place définitive du pouvoir royal à Tonga et le développement des « chefferies " à 'Uvea, vont reprendre et se développer les relations maritimes avec l'extérieur essentiellement en direction de Tongatapu et des archipels périphériques (Vavau, Niua...). L'intensification des relations 'Uvea/Tonga aboutira à l'émergence d'une "chefferie » d'origine tongienne, dans le sud de l'île, autour d'atterrages réputés tongiens dont la tradition orale se fera l'écho, compte tenu du rôle déterminant et des conséquences qu'aura cette implantation dans l'histoire ultérieure de 'Uvea. On a souvent interprété - à tort nous semble-t-il - le développement de cette puissante chefferie (et ce qu'il en est advenu) à travers laquelle 'Uvea « empruntera » à la culture tongienne certains modèles institutionnels, en termes de "colonisation» / " décolonisation », un modèle qui, parce qu'il emprunte à notre propre culture politique, suggère des relations d'un certain type (Sand, 1998 et 1999). La tutelle tongienne, si tutelle il y a eu, n'intègre pas politiquement l'île de 'Uvea dans l'espace tongien. 'Uvea ne constitue pas non plus, à cette époque de son histoire, une entité territoriale ayant une identité et une configuration politique propres. Son organisation s'apparente plutôt à celle d'une confédération de tribus plus ou moins autonomes et antagonistes, plus proche sur le plan du modèle de l'organisation maorie (iwi) ou Fidjienne (vanua) que de la royauté tongienne. Certaines formulations du père Joseph Henquel dans le Talanoa ki uvea nei - influencé par sa propre culture européenne ont aussi beaucoup contribué à conforter par la suite cette image de « domination institutionnalisée » chez beaucoup d'auteurs et certains intellectuels wallisiens eux-mêmes. Pourtant la présentation et l'interprétation de la nature du rapport 'Uvea / Tonga sont généralement beaucoup plus nuancées chez les informateurs comme le montre la transcription de nombreux enregistrements de tradition orale que nous avons effectués autour de ce thème.

12. Nous utilisons ici le terme de lignage au sens large de « groupe territorial de parenté » sans ouvrir le débat sur ce qu'il recouvre exactement («tribus », « lignages », etc.) ou sur la structure de ces groupes (« clan territorial», « ramage », « clan conique » etc.). Voir Paul Kirchhoff (1955) et Marshall Sahlins (1968).

13. Le fatogia consiste non seulement en dons de nattes et de nourritures, mais aussi en prestations de travail. 
- La maîtrise de la navigation

De leur lointaines accointances avec les navigateurs Lapita, les gens de 'Uvea ont su conserver, tout au long de leur histoire, une grande maîtrise de la navigation hauturière, de la construction navale et du savoir qui va avec. Seule l'irruption de la «colonisation » européenne mettra un terme à cette activité et verra la disparition des grands kalia à double coque.(cf. Corpus de traditions orales; Rensch, 1981 : 15-19; Guiot, 2000 : 112-120).

Le contrôle de la navigation paraît avoir joué un rôle non négligeable dans la dynamique d'intégration du " royaume » de 'Uvea. À partir des Takumasiva et au cours du XVII ${ }^{\mathrm{e}}$ siècle, après la consolidation institutionnelle du royaume, les relations transocéaniques en quête de nouvelles alliances vont se redéployer dans toutes les directions (Samoa, Tokelau, Tuvalu, la NouvelleCalédonie (Uvea Lalo), Tikopia, les îles Banks, peut-être aussi Rotuma et Fidji, tout en maintenant avec le royaume de Tonga des rapports privilégiées mais politiquement plus distendues.

En dernier lieu, la venue des Européens et tout particulièrement des missionnaires, bouleversera les équilibres régionaux et la dynamique de toutes ces configurations relationnelles et d'échanges. L'opposition entre missions catholiques et protestantes (Poncet, 1972; Mahina, 1992 ; Angleviel, 1994) remettra en question les relations 'Uvea/Tonga tout comme, à l'inverse, l'accueil de populations catholiques réfugiées en provenance des Tokelau, resserrera en lui donnant un autre sens des liens anciens, réels et/ou mythiques, entre les deux archipels. Mais c'est là une autre histoire (Huntsman, 2004).

Apportons quelques précisions sur l'histoire et la nature même de ces relations à l'époque pré-européenne entre 'Uvea et les archipels environnants :

Avant d'être dominées par Tonga, les relations avec les archipels voisins furent d'abord orientées de façon privilégiée vers les Samoa. Les relations avec cet archipel datent des premiers peuplements. Elles pourraient inclure dans la même orbite des relations suivies avec Futuna. C'est ce que laissent entendre les recherches de linguistique historique sur le proto-polynésien et la classification des langues polynésiennes (Pawley et Green, 1984 ; Pawley et Ross, 1993 ; Kirch, 2001 ). Cette orientation dominante était déjà suggérée par Edwin G. Burrows (1938a et 1940) suite aux travaux de Robert W. Williamson
(1924). Les rapports avec Samoa se seraient ultérieurement distendus, voire interrompus. Par contre, il est probable que les relations avec Futuna aient perduré.

Une tradition recueillie par John B. Stair attribue même le premier peuplement de l'ouest de l'île de Savai'i à des pirogues venues de 'Uvea :

«Pili et ses compagnons qui abordèrent dans la partie ouest de Sawai'i, trouvèrent cette île et 'Upolu déjà occupée et peuplée depuis longtemps. La tradition ne dit pas explicitement d'où ils venaient, mais l'on m'a dit à Sawai'i qu'ils venaient directement de l'île Wallis, ou Uea » (Stair, 1894, cité par Burrows, 1937)

Pour les époques plus récentes, la tradition orale mentionne périodiquement des "alliances » avec des femmes originaires de Samoa ou de Futuna. Certaines lignées ont des attaches avec Futuna, île à laquelle font aussi référence nombre de toponymes ${ }^{14}$.

Les traditions que nous avons recueillies sur les rapports entre 'Uvea et le monde des atolls (Tokelau et Tuvalu) confirment et prolongent celles déjà notées par Joseph Henquel (1910). La participation des deux 'aliki de Fakaofo, Tu'a et Fafie, à la guerre contre Kafoalogologofolau à l'origine du titre de chefferie de Gahi' ${ }^{15}$, kalea, souligne des relations peut-être anciennes avec cet archipel, d'autant que la venue de Tu'a à 'Uvea est rattachée, dans d'autres versions, à l'expédition de Kau'ulufonuafekai et à Havea Fakahau. La tradition des Tokelau est muette sur cet épisode particulier. Fafie, fils de Pio apparaît cependant dans la généalogie qui fonde la « chefferie » de Fakaofo à peu près à la même période. Fafie est aussi connu comme la divinité tutélaire de la navigation et des voyages (MacGreggor, 1937 ; Hunstsman, 2004 ; Huntsman et Hooper, 1996; Huntsman et Hooper, 1991).

Par contre, dans ce même corpus de traditions - ainsi d'ailleurs que dans le Talanaoa ki 'Uvea $n e i$ - il n'est fait pratiquement aucune mention de relations explicites avec l'archipel des Fidji alors que beaucoup d'indices incitent à penser qu'elles ont dû jouer un rôle non négligeable, au moins dans les périodes les plus anciennes, avant la période Atuvalu (voir plus avant, le chapitre "Chronologie et périodisation »). On ne peut, dans le cadre de cet article aborder sérieusement, si ce n'est pour mémoire, le dossier très complexe des rapports entre 'Uvea, le monde mélanésien et

14. Des guerriers futuniens sont mentionnés dans beaucoup de conflits locaux. Il y avait un Futunien dans l'expédition entreprise par Kaukelo à Uvea Lalo (cf. Corpus de traditions orales).

15. Une partie de la population actuelle de Gahi se dit d'origine Tokelau, descendant de Tu'a qui serait resté à 'Uvea alors que Fafie serait retourné à Fakaofo. 
les « outliers » polynésiens, les îles de culture polynésienne différenciée imbriquées dans les archipels mélanésiens. L'étude de ces relations s'inscrit dans une problématique de l'évolution historique de la coupure culturelle Mélanésie/ Polynésie et de ses développements quant à la compréhension du peuplement de l'Océanie insulaire, l'interprétation des données de l'archéologie et de l'anthropologie historique régionale.

Il existe à 'Uvea un certain type de céramiques qui n'entre pas dans le schéma proposé et qui pourrait être intrusif, attestant de relations interinsulaires. La fouille du Malamatagata (MU021) (section 15 en Y 38) a mis au jour un tesson de poterie décoré d'impressions de natte connu aux Fidji, notamment dans le site de Sigatoka (période Natunuku), mais aussi à Futuna (poterie Matakiga II) où elle est datée de $1530 \pm 170$ BP (éch. ANU 9752) dans le site AL 98, Matakiga, à Vele. Cette poterie se trouve également en stratigraphie dans le site SI 70, Fale Toa, à Toloke - Futuna (Frimigacci et Vienne, 2001). Un autre type de céramique atypique décoré de reliefs appliqués provient d'un ramassage de surface à 'Uvea. Cette poterie apparentée au Mangaasi se retrouve, pour la région qui nous intéresse, aux Fidji mais aussi à Futuna, notamment en surface de l'île d'Alofi (un tesson, site AF 43, $\mathrm{Ka}$ tu eni) et en stratigraphie dans les sites de Matakiga à Vele et Fale Toa à Toloke. Cette poterie (Matakiga I) n'est pas datée mais, compte tenu de sa position stratigraphique, devrait être plus ancienne que la poterie décorée d'impressions de natte (Matakiga II) - voir Daniel Frimigacci et Bernard Vienne (2001). Les relations éventuelles avec Fidji, à cette époque, auraient pu s'établir par le biais des relations avec Futuna.

L'étude des relations entre 'Uvea et Fidji demande au préalable une approche analytique comparée plus exhaustive des données empiriques fournies par la recherche archéologique et l'anthropologie des systèmes sociopolitiques décrits par l'ethnographie - que nous ne pouvons entreprendre ici - avant de pouvoir être replacée dans la perspective d'une ethnohistoire régionale. La mémoire traditionnelle de 'Uvea est peu prolixe quand il s'agit de Fidji. On se contentera de souligner la parenté qui semble se dessiner entre le concept Fidjien de vanua et la « chefferie à titre », le groupement politique et territorial de parenté auquel nous faisons allusion sous l'intitulé de « chefferie » indépendante à la période pré dynastique.
La tradition orale à 'Uvea n'évoque guère, non plus, les relations avec Rotuma, anciennes ou plus récentes, pourtant bien attestée par les traditions de Rotuma (Macgreggor, m.s ; Howard, 1985).

Dans l'attente de nouveaux développements de la linguistique et de l'archéologie, l'image que l'on peut dresser des "relations en retour " du " berceau polynésien » vers le monde mélanésien et sa complexité, repose bien souvent sur des faits très ténus, difficiles à réinsérer dans une chronologie régionale. Il est néanmoins probable que ces «Polynésiens extérieurs» établis en Mélanésie proviennent, au moins pour partie d'entre eux, de cette " communauté culturelle » qui s'est différenciée dans le «berceau polynésien » au cours des premiers siècles du premier millénaire B.C. ou du moins qu'ils en sont la composante dominante eu égard aux langues et aux systèmes sociaux.

Les premiers peuplements d'Anuta et Tikopia - peut-être même d'îles de culture mélanésienne comme Pakea aux îles Banks - auraient aussi bien pu s'effectuer à cette période, en partie à partir de Futuna ou de 'Uvea, comme le suggèrent parfois les fragments de silex et vestiges céramiques mis au jour. La présence d'herminettes de Polynésie occidentale à la période a-céramique, et certaines traditions orales attestent aussi de relations à une époque ultérieure (Kirch et Yen, 1982).

Les récits de tradition orale d'Anuta font état d'un peuple ancien envahi par des « étrangers » venus de Uea (Kirch et Rosendahl, 1976). Raymond Firth évoque des traditions du même ordre pour Tikopia ${ }^{16}$. Bernard Vienne, aux îles Banks, a recueilli des traditions, souvent confuses, qui évoquent le passage de «grandes pirogues » en provenance tantôt de Tonga, tantôt de 'Uvea ou de Futuna. Ces récits, à l'exception de celui racontant l'arrivée à une période plus récente de pirogues tongiennes à Pakea, ne font cependant pas référence à une patronymie, une toponymie, des chants ou des textes de littérature orale codifiés par la tradition. En outre, ces îles lointaines sont parfois "confondues» avec Tikopia ou Anuta.

On connaît à Rennell et Bellona la référence à une « terre d'origine » nommée Umbea et certaines traditions de Rennell attribuent même son peuplement d'origine à une pirogue en provenance de 'Uvea (MacGreggor, 1943 ; Hogbin, 1931; Elbert et Monberg, 1965 ; Monberg, 1991).

Le voyage à Uvea Lalo (Îles Loyautés, Nouvelle-Calédonie) que la tradition de 'Uvea

16. " Among the reputed ancestrals lands are Tonga, 'Uvea, Rotuma, Samoa, Taumako, Luangiua (Ontong Java), Pukapuka, Somosomo (Fiji) and Varuka (Banks Islands). » (Firth, $1961: 86$ ). 
attribue à Kaukelo est bien connu. Il aurait eu lieu sous le règne de Lavekava (niveau 6) ou plus probablement de Manuka (niveau 8) ou de Tufele (niveau 7). Notons que cette expédition était sous le commandement d'un chef de pirogue appartenant à la lignée des Takumasiva et que l'équipage comprenait, outre un Futunien, un homme - un frère ou un germain - de la lignée du Kivalu, un de celle du Fotu'atamai, un de celle du Kulitea et un de celle du Pului'uvea, quatre des titres du kau'aliki.

« 125 - C'est à cette époque là que partirent à 'Uvea Lalo Fuimatu de la lignée de Fotu'atamai, Fekai de la lignée de Kulitea et Taginoa de la lignée Kivalu. $126-$ On dit que le hau de l'époque était Lavekava. " (Frimigacci et Pilioko, n.d. : 47).

L'étude comparative de la langue de 'Uvea et du Faka Uvea (Ozanne-Rivierre, 1994 et communication personnelle) ainsi que les aspects mythiques et archétypique du récit suggèrent que cet épisode précis pourrait prolonger des relations antérieures plus anciennes.

\section{Regards sur la chronologie de 'Uvea}

\section{Repères chronologiques}

\section{- Datations archéologiques}

Les sites majeurs de la préhistoire de 'Uvea se trouvent sur la côte ouest et notamment dans le région d'Utuleve dans le sud de l'île où un seul «campement» a pu être fouillé. L'autre site majeur se trouve à Hihifo, toujours sur la côte ouest. Les prospections montrent que celle-ci a été initialement peuplée et peut-être la région nord-nord-est de 'Uvea compte tenu de l'écogéographie (dunes, marécages etc.) plus propice à l'établissement des premières implantations Lapita mais aussi à la préservation des vestiges. La région de Mu'a, au sud, se trouve dans un environnement où le substratum basaltique est très proche de la surface ou même affleure, tandis que le toafa est stérile d'un point de vue archéologique ${ }^{17}$. On trouve encore des jardins humides proches des zones marécageuses. Ces caractéristiques ont rendu très difficile la mise en évidence et la fouille de sites à aptitude humaine.

Dater un site archéologique en Océanie a toujours été un exercice difficile même après les années 1980 qui marquent cependant un progrès notable dans les techniques de laboratoire.
Contamination par les isotopes radioactifs, difficulté d'échapper à la pollution due aux travaux horticoles, rareté $\mathrm{du}$ 《 bon » charbon à remettre au laboratoire, etc. C'est pourquoi, dès les années 1980, Matthew Spriggs (1989) s'était soucié de ce problème. D'autres auteurs, notamment Atholl Anderson (1991), Atholl Anderson et al. (1994), Matthew Spriggs (1990), Matthew Spriggs et Atholl Anderson (1993), Michael Graves et David Addison (1995), vont contribuer ainsi à réfléchir à ces problèmes d'hygiène chronométrique. C'est ainsi qu'un grand nombre de datations, notamment celles des Fidji sont considérées aujourd'hui comme inacceptables. Les travaux récents montrent que ces datations seraient trop anciennes et que la Polynésie occidentale et les îles Fidji auraient pu être peuplées, dans l'état actuel des connaissances, au plus tôt vers 3500 BP (site de Lolokoka, NT-90 à Niuatoputapu, [Kirch, 1988]. Cette datation étant considérée cependant comme acceptable avec réserves (en anglais « questionable ») par Anita Smith (2002: 48), sachant que les autres datations de la zone ne posant pas de problèmes se situent résolument aux alentours de 2800 et 3000 BP (Smith, 2002 : 48, fig. 4-2).

En ce qui concerne la date présumée des premiers peuplements de 'Uvea, que penser de la datation obtenue par l'échantillon de charbon ANU 8442 recueilli dans le site de 'Utupoa ${ }^{18}$ ? Peut-on accepter l'ancienneté du peuplement de 'Uvea aux alentours de 3000 BP (éch. ANU 8442 : $3076 \pm 170$ BP, date calibrée [Stuiver et al., 1998] 2 sigma : 1376/1320 BC) ? Le site de 'Utupoa a été répertorié à l'occasion de l'inventaire de 1982 et 1983 (Frimigacci, Siorat et Vienne, 1983 et 1984). Il se trouve sur l'emplacement supposé, par la tradition orale, d'un habitat aujourd'hui déserté. Trente tessons de poterie de type Utuleve III jonchaient l'emplacement présumé du «village » de 'Utupoa. Un chantier d'une superficie de $15 \mathrm{~m}^{2}$ fut ouvert.

Les trente premiers centimètres avaient été profondément remaniés par les activités horticoles contemporaines. De ces couches remaniées, proviennent 287 tessons dont huit bords, quatre d'entre eux sont décorés d'incisions. Cette poterie épaisse et solide est caractéristique d'Utuleve III. À partir de $30 \mathrm{~cm}$ de la surface on reconnaît la présence de couches en place. À moins $40 \mathrm{~cm}$, sur toute la surface décapée un ensemble de pierres de foyer et sa vidange sont mis au jour sur un sol rubéfié. Les restes de ce foyer apparaissent

17. Espace de collecte, le toafa n'a jamais fait, non plus, l'objet d'une mise en valeur agricole sauf dans la période la plus récente (bananeraies, reforestation, etc.).

18. Toutes les datations discutées ici sont référencées dans Daniel Frimigacci (2000), où elles ont été publiées. Rappelons que les corrections pour l'hémisphère sud ont été effectuées sur les datations non calibrées données par le laboratoire d'analyse. 
dans l'une des coupes du chantier. Cette surface pourrait être une surface d'occupation du village ancien attesté par la tradition orale. Entre -30 et $-40 \mathrm{~cm}$, la fouille a mis au jour 254 tessons de poterie dont 6 bords. Cette poterie s'apparente à de l'Utuleve III. Deux échantillons de charbon relevés à la base du foyer, à $-44 \mathrm{~cm}$, ont montré que la poterie était encore en usage dans le district de Hihifo à Wallis entre 1483 et 1632 de notre ère (dates calibrées, échantillons ANU 8443 et $8443 \mathrm{~B}$, voir supra). À partir de $-40 \mathrm{~cm}$, la poterie de type Utuleve II apparaît, 72 tessons de cette poterie ont été relevés entre -40 et $-50 \mathrm{~cm}$. La couche est bien en place, nous avons trouvé trois raccords céramiques. Des charbons datent cette couche et ce type de poterie aux environs de l'an 1285 de notre ère (date calibrée, échantillon ANU 8441). Plus bas et jusqu'à $-85 \mathrm{~cm}$ de la surface, on observe un remplissage homogène argilo-sableux sans solution de continuité caractérisé par la présence de tessons de poterie de type Utuleve II. À la base de la fouille, à $-85 \mathrm{~cm}$ de la surface, la poterie de type Utuleve I (Lapita pointillé) apparaît, associée à quelques charbons ayant fait l'objet de l'échantillon ANU 8442. Cette datation de $3076 \pm 170 \mathrm{BP}$ se situe dans la fourchette acceptée d'un peuplement de la Polynésie occidentale autour de 2800 et 3000 BP.

Rappelons enfin qu'à partir de $40 \mathrm{~cm}$ de la surface les couches sont bien en place, les niveaux culturels correspondent à la stratigraphie. Les datations ne sont pas inversées et elles correspondent aux vestiges relevés. On observe que la plus grande concentration de tessons de poterie se rencontre dans la couche à $30-40 \mathrm{~cm}$, là où la fouille a mis en évidence des traces d'une éventuelle surface d'occupation (pierres de foyers sur le même niveau et vidange de foyer). Cependant, l'homogénéité de la céramique (de type Utuleve III) récoltée depuis la surface jusqu'à $0,40 \mathrm{~m}$ de profondeur et la forte concentration de vestiges à $40 \mathrm{~cm}$ permettent de penser que les datations de $400 \pm 60 \mathrm{BP}$ et $340 \pm 50 \mathrm{BP}$ (Ech ; ANU 8443 et 8443 B, date calibrée 1483 AD et 1528/1632 AD) (Frimigacci, 2000: tab. 4) obtenues sur les charbons du four en place sont pertinentes pour enfin dater les niveaux les plus récents occupés par les gens de 'Uvea ayant encore l'usage de la céramique. Ces datations du four confirment les autres datations obtenues sur la présence tardive de la céramique à Talietumu (cf. infra: construction du monument Talietumu : éch. ANU $9097: 1126 \pm 60$ BP) et à Utuleve où une autre fouille date cette même poterie de $546 \pm 50$ BP (éch. ANU $9451:$ date calibrée 1407 AD) - (Frimigacci, 2000, tabl. 3 et 2). Cette datation obtenue sur des charbons d'un foyer en place avec de la poterie a été obtenue à la base du niveau d'occupation sur la dune stérile. Ces datations sont les plus récentes obtenues pour la poterie Utuleve III.

La fouille du site HI012 d'Utupoa a permis de dater de $400 \pm 60 \mathrm{BP}$ et $350 \pm 50 \mathrm{BP}$ (éch. ANU 8443 et 8443 B date calibrée 1483 AD et $1528 / 1632 \mathrm{AD}$ ) un foyer en place sur une surface d'occupation bien attestée, caractérisée par de la poterie de type Utuleve III.

Les datations montrent que la céramique est présente à 'Uvea autour de l'an 1000 de notre ère. Enfin les relevés d'anciens villages opérés au cours de notre inventaire avaient déjà montré que la concentration de tessons de poterie en surface était bien plus importante sur les centres présumés des villages qu'à leur périphérie.

La stratigraphie et les datations des sites de 'Utupoa (HI012) et d'Utuleve (MU021) montrent une occupation permanente de la région jusqu'à l'arrivée des missionnaires. L'étude de la répartition des tessons de poterie sur toute l'île indique que les populations porteuses de la poterie de l'Utuleve I étaient installées sur cette côte ouest près des rivages et en face des passes, mais que les porteurs de la poterie de type Utuleve II vont occuper tout le territoire, à l'exclusion du toafa et du vao tapu (la région forestière de la partie sud de l'île). Plus tard, les porteurs de la poterie de type Utuleve III vont s'installer partout également mais d'abord dans le sud et plus tardivement au nord, réserve faite du toafa où l'on n'observe aucun vestige d'occupation humaine permanente.

La construction des monuments se serait effectuée en deux temps. Le premier coïncide avec les débuts de la période Atuvalu et l'apparition de la céramique de type Utuleve III vers les années 1000/1200 de notre ère. Ceux de la deuxième phase, encore visibles aujourd'hui, coïncideraient avec la présence des Tongiens rapportée par les traditions orales, et débuterait vers les années 1300/1400. 
À $45 \mathrm{~cm}$ de la surface de la plate-forme nordouest de la résidence du Talietumu (MU029), ont été mis au jour des vestiges d'une plate-forme ancienne avec un foyer en place, datée de $1126 \pm$ 60 BP (échantillon ANU 9097, soit date calibrée de : 898/944 AD). Dans le foyer, on note la présence de la céramique de type Utuleve III. C'est bien cette poterie qui a été datée dans ce foyer, car immédiatement au-dessous on rencontre le substratum basaltique bien en place. Cette datation, la plus récente pour ce type de poterie, marque le début de la période Atuvalu. Elle semble fiable car le foyer daté se trouvait bien protégé sous le monument actuel.

La construction du monument de Malamatagata (MU021) se serait également opérée en au moins deux temps : la première remonterait à la période Atuvalu, vers l'an 1281 de notre ère.

Un autre monument ancien (période Atuvalu) non daté a été mis au jour sous la résidence dite du Kalafilia située à proximité du Malamatagata. Il est constitué de blocs de basalte emballés par endroits dans des éléments divers, notamment du corail, des sédiments argilo-sableux et des galets de plage. Les sondages effectués entre la plage et le monument ont fait apparaître les cuvettes dont le sable aurait été extrait. Un foyer en place (Frimigacci, 2000 : fig. 13 - Malamatagata, section 15 Y 33), situé immédiatement sous le monument initial et renfermant de la poterie de type Utuleve III a été daté de $736 \pm 60 \mathrm{BP}$ (date calibrée : 1281 A.D. échantillon ANU 10071). Une autre datation, obtenue à partir de charbons prélevés dans le remplissage du premier monument, est plus ancienne que le foyer en place $(1166 \pm 80 \mathrm{BP}$ date calibrée : $888 \mathrm{AD}$, échantillon ANU 10072) : elle date les sédiments qui ont été prélevés pour la construction du monument.

La sépulture de Puhi (voir plus avant $\S$ Les acteurs de l'histoire) permet de dater la fin de la période Atuvalu aux alentours de 1300 1400 A.D (ANU 7394A, $670 \pm 200 \mathrm{BP}$; ANU 7394B, $560 \pm 100 \mathrm{BP})$. Les fouilles de la sépulture collective de Petania (HI015) et du tertre funéraire de Pelapela (HI024) n'apportent pas d'informations déterminantes pour notre propos dans la mesure où leur attribution reste sujette à caution.

Les aménagements horticoles et en particulier les grandes tarodières irriguées de Vailala (HI011), de Falaleu (HA010) ou de Nuaga (MU090) qui ont été relevées n'ont pu être datés.

\section{- Niveaux généalogiques}

La chronologie par niveaux généalogiques, ici utilisée, n'est pas à proprement parler une chro- nologie mais plutôt un système de coordonnées, une matrice, qui permet de positionner, les uns par rapport aux autres, événements et personnages. C'est seulement en tant que totalité structurée qu'elle prend une valeur chronologique. Les mariages dessinent les articulations principales entre les lignes généalogiques recalées entre elles par le biais de la " contemporanéité ».

La matrice de notre chronologie par niveau généalogique considère l'année 1980 comme point zéro de la chronologie. Nous comptons 28 ans par niveau généalogique. Le niveau généalogique zéro est donc, en principe, celui des personnes nées entre 1952 et 1980 . On ne saurait cependant établir une concordance étroite et directe entre le niveau généalogique relatif des personnes et leurs âges relatifs. Deux personnages situés au même niveau généalogique peuvent présenter une différence d'âge de plus d'une génération comme des personnages contemporains peuvent être séparés par un, voire deux niveaux généalogiques. La polygamie qui accentue la différence d'âge entre les conjoints et entre les " pères » et les «fils"-tout particulièrement pour ceux de statut social élevé - est un facteur qui se surajoute à toutes les «manipulations » éventuelles du « tissu » généalogique mémorisé, pour potentialiser l'effet de "décrochage» des niveaux généalogiques les unes par rapport aux autres et par rapport au temps comptabilisé. L'« adoption » en est un autre. C'est souvent le niveau de la segmentation entre lignées collatérales qui se trouve ainsi « escamoté » quand l'on veut se rapprocher généalogiquement d'un ancêtre prestigieux ou référent d'une légitimité à faire valoir. Un niveau peut également se trouver « omis » du fait qu'il serait représenté par quelqu'un qui n'a eu aucun rôle prépondérant dans l'histoire. C'est ainsi que l'on observe dans le cas qui nous intéresse une discordance singulière dans le raccordement de la généalogie de Takumasiva avec celle de Vehi'ika qui chacune pour elle-même présente une très grande cohérence interne.

La tradition orale qui se rapporte à la tradition antérieure à la dynastie des Takumasiva ne renvoie pas, elle, à une structure généalogique cohérente. De plus un examen attentif du texte de référence montre qu'il n'y a pas de continuité dans la structure du récit traditionnel entre ce qui se rapporte à la période des forts et ce qui se rapporte à la période dynastique. Il apparaît que le père Joseph Henquel, et peut-être même ses informateurs, se soient efforcés de rattacher à la tradition dynastique un corpus de traditions dont la diachronie procède d'un autre schéma d'interprétation que celui centré sur la transmission généalogique du hau. Il semble bien que les 
événements rapportés pour cette période de l'histoire de 'Uvea se soient déroulés, pour autant qu'ils aient bien eu lieu, pendant une période relativement brève, en l'espace des quelques décennies qui séparent dans la chronologie tongienne le règne de Kau'ulufonuafekai de celui de 'Uluakimata dit Telea, soit deux, trois au plus, niveaux généalogiques. La figure 1 présente le cadre de la chronologie généalogique que nous avons retenu pour la restitution de l'histoire pré européenne de 'Uvea' ${ }^{19}$.

\section{- Chronologies régionales}

Le recours aux chronologies régionales, séquences archéologiques et chronologies généalogiques, est particulièrement utile dans le cas de 'Uvea du fait des relations culturelles privilégiées, à l'origine, avec Samoa et de la continuité des relations avec Tonga qui fournit ainsi un « cadre de référence » à la tradition orale, puisque les traditions orales tongiennes constituent, au regard de la chronologie, l'un des corpus les plus détaillés et les plus fiables. La figure 2 présente la chronologie généalogique de 'Uvea par rapport à celle établie pour Tonga et Futuna.

\section{Les acteurs de l'histoire}

Les principaux « personnages » de l'histoire à tradition orale de 'Uvea nous sont connu, non seulement pour ce qu'en dit la tradition mais par leurs résidences, leurs sépultures, ou encore les vestiges relevés auxquels on peut les associer, ainsi que par des traditions localisées liées au foncier et à la toponymie ${ }^{20}$.

Tagaloa et Maui. Dieux, demi dieux ou héros de la mythologie polynésienne, c'est selon, ils sont aussi invoqués à 'Uvea. La cosmogonie de 'Uvea est cependant plus proche de celle de Tikopia que des cosmogonies sophistiquées de la Polynésie centrale et orientale et du monde Maori.

« Traditionally Tikopia seem not to have possessed any cosmogony of a very general kind, dealing with the origination of the universe, or of the world, far beyond their own island home [...] Tikopia traditional tales then are egocentric and uninquisitive. They are concerned with conditions in their own land, and in their society and they show no interest in the origins of other lands and people. » (Firth, 1961: 21-24)

Elle est aussi moins élaborée que celles de Samoa ou de Tonga (Mead, 1930; Gifford 1929). Centrée sur la seule île de 'Uvea, elle se préoccupe peu de justifier la genèse du monde insulaire environnant que l'on tient pour acquis. Elle fait toutefois référence aux héros civilisateurs et demi-dieux que sont Tagaloa pour la création de l'île et Maui ${ }^{21}$ pour sa " découverte».

La création des hommes à partir du fue (Canavalia maritima), qui engendre les hommes et les femmes sous la forme de vers, relie Tagaloa à Maui en utilisant un schème assez courant :

«11 - Tagaloa aurait envoyé sur terre sa fille Hina avec un fue (Canavalia maritima). Arrivée sur terre, Hina plante son fue pour s'abriter du soleil. Hina repart. Longtemps après, elle revient voir sa plante, mais celle-ci est morte. À sa place Hina trouve des vers. Elle s'en va de nouveau puis redescend un peu plus tard sur terre pour constater que les vers se sont changés en hommes et en femmes.» ${ }^{22}$

Le père Joseph Henquel rapporte aussi une autre tradition, un thème d'ailleurs commun à la plupart des îles de la Polynésie, de Tonga à Tahiti, dans laquelle Maui Atalaga repousse le ciel de la terre, tenus séparés seulement par le mahoa (Tacca leontopetaloides) et le teve (Amorphophalus campalunatus), permettant alors aux hommes de se redresser. Le mythe d'origine du titre de hoko ${ }^{23}$, descendant du couple Fatuloamaka et Fuila'oa, se revendique, lui, de cette tradition (Burrows, $1937: 162 ; 1945: 57$ ).

Hauolekele, Ufi et Lupelutu. Ces trois patronymes - supposés venir de Tonga - sont mentionnés comme l'origine de la population de 'Uvea par le Talanoa $k i$ 'Uvea nei. On ne les retrouve pas mentionnés dans la tradition tongienne et on ne leur connaît pas de filiation ultérieure à 'Uvea.

« However, there is also evidence of a pre-Tongan population and of immigrations from other islands. Henquel (p. 18) says the population of Uvea was

19. Ce tableau généalogique ayant pour seul objectif de fournir et justifier un cadre chronologique ne prend bien entendu pas directement en compte l'ensemble des données généalogiques dont nous disposons pour cette période.

20. Nous renvoyons ici à l'inventaire systématique que nous avons effectué dans les années 1980 qui fît l'objet de plusieurs rapports partiels (par ex. Frimigacci et alt., 1983 et 1984) publiés au centre ORSTOM de Nouméa (IRD) et dont l'édition définitive est aujourd'hui envisagée.

21. Ici Tagaloa ’i Lagi et Maui Atalaga accompagné de son fils Maui Kisikisi (Burrows, 1937 : 161)

22. Notre traduction (Frimigacci et Pilioko, n.d.).

23. Le titre de Hoko apparaît dans la tradition orale de 'Uvea comme le titre le plus ancien - hors chronologie. Il a aussi une fonction rituelle : celle d'être l'intronisateur au titre et aux fonctions de $h a u$, du moins dans la période prédynastique de l'histoire de 'Uvea. Ce rôle sera ensuite assumé tantôt par le mahe, tantôt par kivalu, ou encore par Kivalu et Kulitea. C'est aujourd'hui le titre de chefferie de Ha'afuasia. Il ne joue plus aucun rôle rituel ou cérémoniel particulier ni dans la cérémonie d'intronisation au hau, ni dans celle de kava, ni même dans les katoaga, les distributions cérémonielles de vivres. 


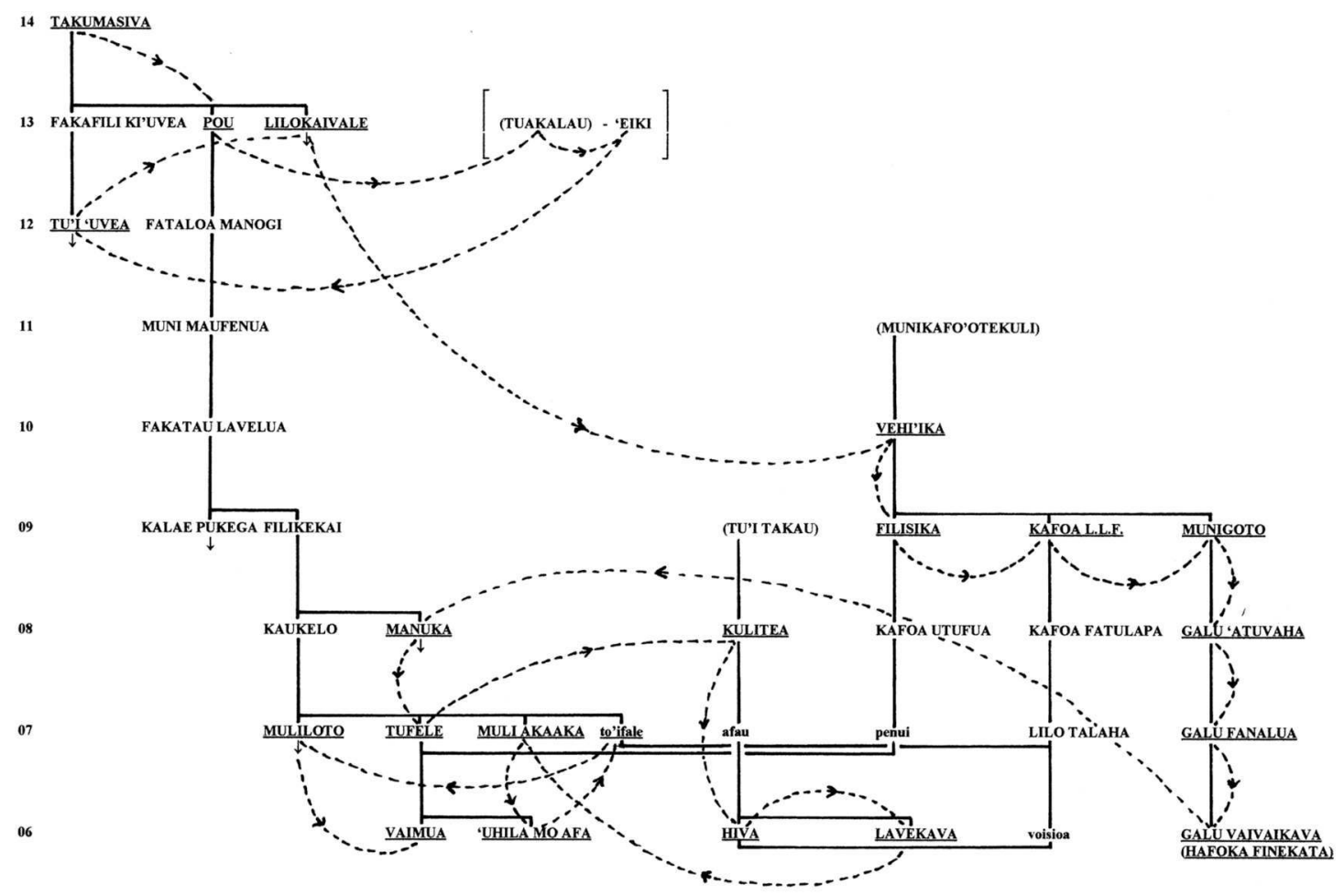

Légendes

$\ldots \rightarrow$ Succession au hau $00 \quad$ Niveau généalogique $\quad \downarrow \quad$ Mariage

FIGURE 1. - ’Uvea : chronologie généalogique.

\section{Niveaux}

Tonga

(Hors chronologie)
Uvea

Futuna

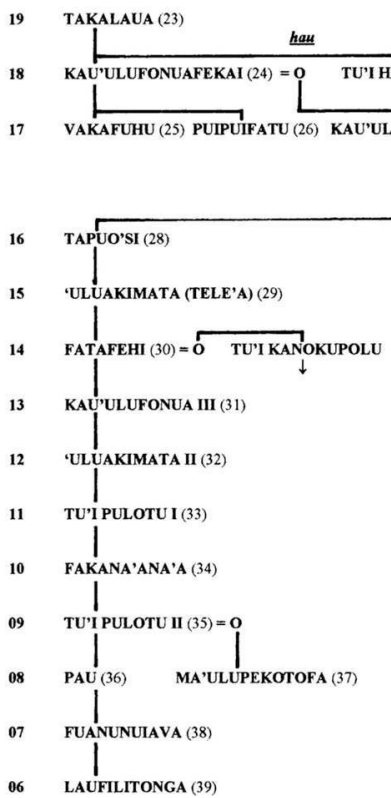

(GA'ASLALILI)

(GA'ASIALILI)

HAVEA FAKAHAU FAKAHEGA

SAKUMANI

MAU'IFA
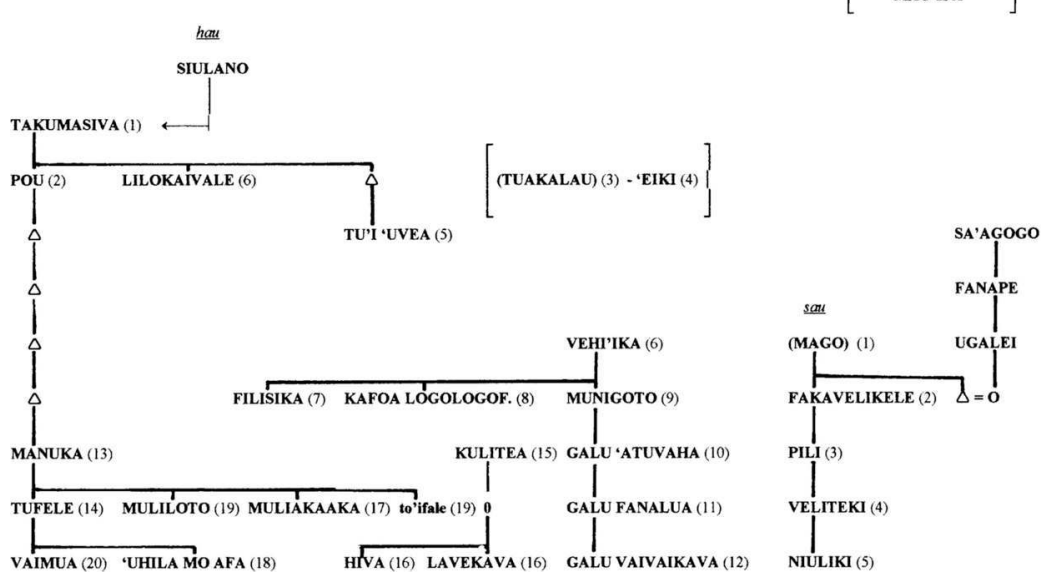

FIGURE 2. - Esquisse d'une chronologie généalogique régionale. 
begun by three immigrants, Hauolekele, Ufi, and Lupelutu. In the copy of his history that I used, he had written in the margin "from Tonga", but gave no evidence to support this statement. I have been unable to find these names in Tongan tradition. Other early Uvean names, not recorded in Tonga, are Tauloko, the first Uvean king, Fatuloamaka, legendary ancestor of the Hoko line, and the chiefs Kalafilia and Fakate; but the present holder of the title Hoko told me that his lineage was "not Tongan, but Uvean" ; and an old song traces the Hoko line to Fatuloamaka. » (Burrows, 1937: 170)

Puhi. Hors niveau généalogique, Puhi nous est connu par un chant très ancien, recueilli par Edwin G. Burrows (1945 : 10), qui en fait mention : il l'associe à la déesse Kakahu ${ }^{24}$ et situe sa sépulture à Atuvalu (MU020), au sommet de la pointe de Lausikula :
«Lausikula mo te 'Atuvalu
Lausikula et Atuvalu
Ko te fakatahi'aga 'o te hau
C'est le lieu pour le rassemblement des hau
Faitoka 'o Puhi mo Kakahu
La sépulture de Puhi et Kakahu
Ko Vaitutulu' 'e ka to
Que vienne le temps de la profanation
Fakaholofagona kua hopo
L'un après l'autre ceux qui sont venus là vont se dresser contre elle ${ }^{25}$ »

La fouille d'Atuvalu (Frimigacci, 1997 et 2000 ; Frimigacci et Vienne, 2001) date la sépulture de Puhi entre 1300 et 1400 AD (ANU 7394A, $670 \pm 200$ BP - ANU 7394B, 560 \pm 100 BP). Quelques générations seulement séparent donc Puhi de Ga'asialili.

Tauloko (niveau généalogique 18 ou 19). Il appartiendrait selon le père Joseph Henquel (Henquel, 1910) au lignage tongien Tu'uhoko. On pourrait en effet être tenté d'en faire un émissaire du Tu'i Loloko, le premier lignage du Falefa du Tu'i Tonga dont le premier matapule à l'époque de 'Aho'eitu s'appelait Tu'uhokokilangi. C'est à notre connaissance la seule référence possible à un lignage tongien. Il a résidé à Ha'afuasia (HA058) (Hava'iki) et y fut enterré. Il a reçu le hau de Hoko. Si l'on suit la tradition déjà évoquée, Hoko serait le premier chef éponyme de 'Uvea, une revendication d'autochtonie et de rang - qui trouve son sens sur le plan rituel et cérémoniel - si ce n'est d'ancestralité proprement dite.
"Henquel says that Hoko was "of the Tui Tonga family called Tuuhoko"; but the present holder of the title Hoko told me that his lineage was "not Tongan, but Uvean" ; and an old song traces the Hoko line to Fatuloamaka. According to Henquel, Ga'asialili on his arrival from Tonga named three chiefs for Uvea: Hoko, Kalafilia, and Fakate. But the chiefs of the Tongan lineages he brought with him were Kula of the Ha'amea, and He of the Ha'avakatolo. Possibly Hoko, Kalafilia, and Fakate were chiefs of a pre-Tongan population » (Burrows, 1937 : 170-171).

La primauté et le rang qui lui sont accordés par la tradition orale ne reflètent peut-être, à tout bien considérer, que l'importance accordée ultérieurement à cette première tentative d'implantation tongienne avec la complicité du Hoko, avant la politique d'expansion territoriale initiée par Takalaua et poursuivie par Kau'ulufonuafekai. En tout état de cause, l'institutionnalisation du hau, comme délégation d'autorité à l'origine de la dyarchie du pouvoir politique à Tonga (Gifford, 1935 ; Bott, 1982 ; Kirch, 1984 ; Douaire-Marsaudon, 1998), lui serait ultérieure. On ne sait s'il faut considérer Tauloko comme un 'eiki tongien, un « chef » à qui est déléguée l'autorité du Tu'i Tonga, ou comme un 'aliki, certes d'ascendance tongienne, mais simple «leader» de l'une de ces « chefferies » du sud, centrée sur la région de Ha'afu'asia.

Hoko, Kalafilia et Fakate (niveau généalogique 17 ou 18). Ce sont les trois premiers titres de chefferie qui sont apparus au tout début de l'historiographie de 'Uvea. S'ils ne sont plus aujourd'hui que de simples titres de chefferie de village, ils ont joué un très grand rôle dans les débuts de l'histoire de l'île.

Ga'asialili - Elili dans la tradition tongienne (niveau généalogique 17 ou 18). Il serait originaire de Tonga et appartiendrait au lignage Tu'i Ha'atakalaua selon Joseph Henquel (Henquel, 1910). D'après la tradition tongienne, Ga'asialili ainsi que Fakahega seraient deux " chefs de famille » du lignage Ha'a Motuapuaka (Gifford, 1925 : 40). Envoyé après la mort de Tauloko, accompagné de guerriers du lignage Ha'avakatolo, sous l'autorité de $\mathrm{He}$, et Ha'amea sous celle de Kula ${ }^{26}$, Ga'asialili ne resta que peu de temps à 'Uvea et s'établit ensuite à Futuna. Il construisit le fort de Kolofau sur l'île d'Alofi. Tué à la bataille de Pakafu (royaume de Sigave) lors

24. Connue à Futuna sous le nom de Finelasi, elle joue un rôle lors de la venue de Kau'ulufonuafekai et/ou Telea (Frimigacci, 1990).

25. Litt. « L'eau s'égouttera de la conduite / L'un à la suite de l'autre ceux qui sont venus là vont se dresser ». L'image de la conduite laissant « pleuvoir l'eau » est une métaphore de la profanation du sacré (Bataillon, 1932 : 390).

26. Le manuscrit du père Joseph Henquel ajoute que ces deux guerriers étaient sous la tutelle d'une femme nommée Lupe Fugakolo. 
d'une tentative d'invasion de l'île de Futuna, il serait enterré à Futuna, à Malae Vaka (SI057).

Havea Fakahau (entre les niveaux généalogiques 16 et 18). Appartenant à une branche du lignage Ha'avakatolo, une branche du Ha'a Ngata Motua, il résida à Ha'afuasia (HA058) Niuvalu, puis à Lalokea, où il aurait été chassé par les frères Talapili et Talamohe. Il fut longtemps au pouvoir, soixante ans dit-on. Mort à Lalokea (HI003, Hihifo), son corps aurait été envoyé à Lausikula, Atuvalu (MU020) ${ }^{27}$ pour y être inhumé. On dit que la grande pirogue Lomipeau, pour autant qu'elle fasse référence à un événement historique précis, aurait été construite sous son règne. On observe sur le 'api Lalokea (HI003), un tertre funéraire ovale élevé, d'une envergure de $17 \mathrm{~m} \times 11 \mathrm{~m}$, sans trace d'appareillage, recouvert de sable blanc, qui pourrait également lui être attribué.

Talapili et Talamohe. Fils de Vaka'ana dont le père Tupa Totolo est un descendant du ramage Ha'avakatolo, ils résidaient respectivement à 'Utufua (MU072) et à Tala'amoa (MU104). Talapili serait enterré à Talamoa et Talamohe à Niuvalu (HA004). On leur attribue un long règne et leur mort serait liée à un conflit avec Toamisa, l'un des fils de Fakahega.

Fakahega. Il serait comme Ga'asialili d'origine tongienne, du lignage Ha'amotuapuaka. La tradition orale de 'Uvea recueillie par le père Joseph Henquel en fait le « successeur » des frères Talapili et Talamohe. Elle l'associe également au règne de Kau'ulufonuafekai. Il aurait vécu à Tua où il pourrait être inhumé, bien que sa sépulture n'ait pas été répertoriée. Marié à Lupeolo, fille du Tu'i Ha'amea, Papa, il se réfugia dans le toafa de 'Afekafu pendant la guerre avec Kau'ulufonuafekai qui libéra 'Uvea de la tutelle politique tongienne. Son fils Puakavahe faisait partie de la flotte qui vint à 'Uvea avec Kau'ulufonuafekai. On connaît les descendants de son fils Tu'i Tafuma.

Tu'i 'Alegau (entre les niveaux généalogiques 16 et 19). Il s'agit du titre ancien d'une chefferie autonome du sud-ouest de l'île. C'est un personnage important des débuts de l'histoire de 'Uvea. Le Tu'i 'Alegau entra en conflit avec les frères Talapili et Talamohe. Ses forces se firent massacrer à Utuleve. C'est Laukua'ulu, le Tu'i 'Alegau dont la naissance à Malama Tagata fut, d'après la tradition, éclairée par des hommes décapités transformés en torches humaines, qui présida au règlement définitif de la guerre du
Molihina en autorisant le mariage de sa fille Simuoko avec Laupuatokia. Ce titre a aujourd'hui disparu.

Maufehi, Laupuatokia et Simuoko - Ce sont les principaux protagonistes de la guerre dite du Molihina (niveaux généalogiques 16 à 14) que nous verrons ci-dessous.

Siulano (niveau généalogique 15). Il résida à Matagaika Lavengahau (MU126), dans le fort construit au sommet d'un promontoire dominant la mer par Munilavengahau et Fakakava son fils, à une époque antérieure. Rapidement destitué du hau par Fakafiliki'uvea et Pou, les fils de Takumasiva, il fut enterré à Matagaika.

Takumasiva (niveau généalogique 14). La tradition est muette sur les origines de Takumasiva, l'ancêtre fondateur de la lignée régnante actuelle et du " royaume » de 'Uvea. On sait seulement qu'il fut porté au titre de hau suite à la destitution de Siulano par ses deux fils : Fakafiliki'uvea et Pou. Certains suggèrent que Takumasiva pourrait être un fils de Siulano, d'autre pensent qu'il serait d'origine tongienne. Plus vraisemblablement, il ne serait que l'aîné d'une des lignées dominant la scène politique de l'époque ${ }^{28}$. On ne connaît ni sa résidence, ni sa sépulture. On sait seulement qu'il fut un « bon » hau.

Pou (niveau généalogique 13). Fils de Takumasiva, il fut assassiné par Tuakalau et Heiki qui usurpèrent le hau. On ne sait où était établie sa résidence ni où il fut enterré. On connaît néanmoins sa descendance jusqu'à aujourd'hui.

Tuakalau et 'Eiki (niveau généalogique 13). Tuakalau et Eiki n'appartiennent pas stricto sensu à la dynastie de Takumasiva sauf à supposer une relation d'alliance matrimoniale qui les aurait intégrés dans le kaiga des Takumasiva. L'épisode suggère une forte tension politique entre les Takumasiva et les chefferies locales plus directement inféodées au Tu'i Tonga. Tuakalau reçut le hau de Mahe. 'Eiki assassina Tuakalau et se proclama hau. Il fut à son tour tué par les hommes du Tuakalau lors de l'affrontement provoqué par Mahe à Tepa (Ha'afata). Tuakalau fut enterré à Malumoehau (MU028).

Mahe (niveau généalogique 13). Il apparaît une première fois dans l'histoire de 'Uvea comme l'instigateur de la tentative d'usurpation du pouvoir par Tu'akalau et 'Eiki. C'est le père de Maupa, la mère de Vehi'ika. C'est lui qui ordonna l'assassinat de Lilo Kaivale et porta Vehi'ika au pouvoir. Le titre de Mahe est encore l'un des titres du kau'aliki.

27. La sépulture d'Atuvalu ouverte et détruite par les missionnaires à la fin du XIX ${ }^{\mathrm{e}}$ siècle lui est depuis attribuée. Dans les témoignages, il y a néanmoins une évidente confusion entre l'excavation de cette sépulture et celle de Vehi'ika (1896) à Talikata, qui a beaucoup marqué les esprits (Poncet, 1972).

28. Il pourrait exister un lien entre Takumasiva et le Kalafilia et/ou le Tu’i ’Alegau (cf. Corpus de traditions orales). 
Tu'i 'Uvea (niveau généalogique 12). Les Takumasiva reprirent le hau avec Tu'i 'Uvea, le fils de Fakafiliki'uvea frère aîné de Pou. Il résida à Hihifo, à Kala'evavaka qui devint Vaitupu (HI055T) et fut enterré à Tafatu'u (HI014). La tradition a retenu son voyage à Vaitupu, aux Tuvalu $^{29}$. Il en aurait rapporté le tuluma (boîte à hameçon) ainsi que le cochon jaune (puaka kelo) et le cochon noir (puaka 'uli). À Rotuma, on parle aussi de la visite d'un Tu'i 'Uvea qui pourrait être ce même personnage.

Lilokaivale (niveau généalogique 13). C'est le troisième fils de Takumasiva, Lilokaivale qui succéda à Tu'i 'Uvea. Il résida à Niuvalu (HA004) et fut assassiné sur ordre de Mahe par Vehi'ika, Kahofuna et Muli'italo. Il fut enterré à Niuvalu (HA004).

Vehi'ika (niveau généalogique 10 ou peut-être $11)^{30}$. Fils de Munikafo'otekuli, un « chef » tongien, et de Maupa, la fille de Mahe, il résida à Finekata (MU025) et Mala'efo'ou (MU071) où il fut enterré31. Les porteurs du titre de chefferie de Kalafilia disent descendre de son fils Kolokitua $^{32}$. Le Tu'ihoua de Tepa, le Gata de Ha'atofo et le Takala de Lavengahau descendraient aussi des fils de Vehi'ika, respectivement de Tu'ihoua, Makalo et Takala. Bien qu'aucun conflit ne soit spécifiquement attaché à son règne, on lui prête les qualités d'un grand guerrier ('aliki toa).

Filisika (niveau généalogique 9). Troisième fils de Vehi'ika, il résida à Te'esi et à Gahi et il fut enterré à Talikata (MU089). Avec l'entregent du Fotu'atamai, il renoue et resserre les liens avec le Tu'i Tonga. Il a la réputation d'avoir été un «bon» hau.

Kafoa logologo folau (niveau généalogique 9). À l'inverse de son frère Filisika auquel il succèda, il fut un hau arrogant, cruel et belliqueux. Il résida à 'Utufua et Niuvalu (HA004) (Ha'afusia) où il fut enterré. À Kafoa, il affronta une coalition menée par Kalafilia à laquelle participèrent Sila et Tua, un 'aliki originaire des Tokelau (Fakaofo) établi à Gahi. Il fut défait. Sa mort signa, dit-on, l'arrêt du «cannibalisme» à 'Uvea.

Munigoto (niveau généalogique 9). A la disparition de Kafoalogologofolau, son frère, Munigoto, reçut le hau. Il résida à Talikata (MU027), Mala'efo'ou et fut inhumé à Talikata ; sa sépulture n'a pas été répertoriée.
Galu'atuvaha (niveau généalogique 8). Il résida à Talikata (MU089) et y fut enterré. Marié à Filifua Takailuatau, fille de Moe'ao, il est le père de Puakauhinima, le premier Kivalu. Il a la réputation d'avoir été un « bon » hau.

Galu Fanalua (niveau généalogique 7). Il résida et fut enterré à Talikata (MU089), Ha'atofo (MU074T). Il se maria à la sœur de Na'atokai, un "chef » tongien, ainsi qu'avec Seutatia, une femme originaire des Samoa.

Galu Vaivaikava et Hafoka Finekata (niveau généalogique 6). Il succéda à Galu Fanalua, résida à Finekata (MU025), Mala'efo'ou (MU071) et épousa Kalae. Il partagea le pouvoir avec Hafoka Finekata à qui il délégua le hau sur le sud-ouest de l'île, de 'Ahoa jusqu'à Ha'alalo. On le dit «méchant» et « incompétent ». Il ne s'occupait pas des affaires du pays et c'est en réalité le Kivalu qui assuma le pouvoir et exerça l'autorité. Selon certains, Hafoka Finekata serait un descendant de Filisika. Cette délégation de pouvoir, de la part de Galu Vaivaikava à une lignée collatérale sur une partie du district de Mua, peut-être bien orchestrée en sous main par le Mahe et le Kivalu, pourrait résulter d'un compromis - pour éviter une partition territoriale visant à réduire les tensions entre les deux lignées collatérales des descendants de Vehi'ika, l'une dominant le sud-ouest du district de Mua, liée au Kalafilia, l'autre l'est et l'ensemble Hihifo Hahake, liée au Mahe.

Kivalu et le $\boldsymbol{k a u}$ 'aliki. C'est aujourd'hui le deuxième titre par ordre de préséance après le Lavelua. C'est le chef de l'exécutif et, malgré son importance, le titre est récent. Le premier Kivalu est apparu dans l'histoire de 'Uvea sous le règne de Galu Vaivaikava. Il s'agirait de Puakauhinima, qui résidait sur l'îlot Nukuione d'Hahake, fils de Moe'ao, petit-fils de Lemanahalatai, dont la sépulture se trouve sur l'îlot Nukuloa (MI009). Sa sœur est l'épouse de Galu'atuvaha. Il aurait joué un rôle politique de premier plan. Il serait enterré à Tokatafa (HA035). C'est à cette période (niveaux 9 8) qu'on a vu se formaliser le $k a u$ 'aliki sous sa structure actuelle. Le kau 'aliki, conseil politique et exécutif du Lavelua, est constitué par les titres auxquels sont associées des fonctions. La hiérarchie des titres au sein du kau 'aliki, de même que le rang et les fonctions symboliques et d'autorité qui leur sont attachés, semblent avoir quelque peu "varié »

29. Pour d'autres, il s'agirait de l'île de Rotuma ou encore de 'Eua à Tonga (Burrows, 1937).

30. En effet, la mère de Vehi'ika est une fille de Mahe (niveau 13) - voir plus haut.

31. Sa tombe fut détruite en 1896 et les dalles réutilisées pour la construction de l'autel de la chapelle de Lanutuli (Poncet, 1972: 43).

32. Selon d'autres versions de la généalogie, Kolokitua serait le fils de Manuisia, le frère aîné de Vehi'ika (cf. Corpus de traditions orales). 
dans le temps et en fonction des conjonctures et de la personnalité des titulaires. Le kau 'aliki est aussi le symbole, sur le plan politique, de l'intégration territoriale de 'Uvea.

Manuka (niveau généalogique 8). Il résida à Hahake, Mata 'Utu (HA008), Mala'e Vaka (HA003). On ne connaît pas avec certitude l'emplacement de sa sépulture. C'est lui qui fut choisi au titre de hau, au lieu de Filikekai chassé de Niuvalu par Hoko suite à un inceste. Pendant son règne eut lieu une nouvelle confrontation, la guerre de Tepa, avec une coalition de Mu'a.

Tufele (niveau généalogique 7). Fils de Manuka, il résida d'abord à Mata 'Utu (HA008T) puis à Talikata (MU089), à Lele'alulu. Ayant décidé de transférer à nouveau la résidence royale à Mata 'Utu, il dressa contre lui les chefferies de Mu'a qui montèrent un complot contre lui auquel participa son demi-frère Muliakaaka.

Kulitea (niveau généalogique 8). Il fut intronisé par une décision du kau 'aliki (Kivalu, Mahe, Fotu'atamai, 'Ului Monua) alors qu'il était déjà âgé. Il résida à Liku, Pakafu (HA045) et fut enterré à Liku, Fakafu (HA025). On dit que ce fut un roi avisé.

Hiva et Lavekava (niveau généalogique 6). Petits-fils de Kulitea, ils prennent le titre de hau à sa suite. Ils furent successivement assassinés par Muliakaaka. Hiva résida à Hahake et Lavekava à Mua. Nous n'avons pas identifié leurs sépultures.

Muliakaaka (niveau généalogique 7). Il résida à Mua mais sa sépulture n'est pas non plus identifiée. Avec l'assassinat de Lavekava et Hiva, il s'empara du hau et épousa Vakapuleia, la fille de Lavekava. Sa conduite dressa contre lui l'unanimité des chefs qui décidèrent de l'éliminer et d'introniser comme hau 'Uhilamohafa.

'Uhilamoafa (niveau généalogique 8). Fils de Tufele, il résida à Hahake et fut enterré à Tokatafa (HA035). Durant son règne qui fut de courte durée, il épousa Luia, la fille de Kae, puis Faufo'o, sœur de Ta'ofifenua de la lignée du Kivalu.

To'ifale (niveau généalogique 7). Première femme régnante ${ }^{33}$, elle mourut en 1829 . Descendante en ligne directe de Takumasiva, elle est la fille de Kaukelo, le frère de Manuka. Elle épousa Lilotaloaha, un petit-fils de Kafoalogologofolau, descendant en ligne directe de Vehi'ika. C'est elle qui portait le hau en 1825 lorsque le capitaine Moane à bord du Mahulelagi accosta à 'Uvea en provenance des îles Hawai'i, suivi par le débarquement et l'installation en 1829 de Siaosi
Manini et de ses marins hawaiens. De père espagnol et de mère hawaienne, Siaosi Manini faisait le commerce des holoturies pour le compte d'Aluhi, un riche commerçant hawaien d'Ohau. Il ouvrit un commerce à 'Uvea et tenta, allié avec Takala, son beau père et descendant de Vehi'ika, de prendre le contrôle politique de l'île.

Muliloto (niveau généalogique 7). Il résida à Ha'afuasia et fut enterré à Tokatafa (HA035). Fils de Kaukelo, le frère de Manuka, il succèda à la reine Toifale. Très âgé, il décéda dans l'année de son intronisation (1829) laissant le hau à Vaimua, connu aussi son le nom de Soane Patita Lavelua.

Vaimua - Soane Patita Lavelua (niveau généalogique 6). Il avait trente ans, en 1829, lorsqu'il accepta le hau. C'était le hau de 'Uvea à l'arrivée des missionnaires en 1837. Il mourut en 1858 et fut enterré au cimetière chrétien de Mata 'Utu.

\section{Les événements fondateurs retenus par la tradition orale}

\section{- Le partage territorial de 'Uvea}

Le père Joseph Henquel situe le partage territorial de 'Uvea entre Hoko, Kalafilia et Fakate à l'époque de Ga'asialili :

« 21 - À cette époque, les trois chefs de 'Uvea étaient Hoko, Kalafilia et Fakate. 22 - Ces trois chefs entreprirent de se partager l'île. Ils se rendirent pour cela à Lanutavake, au pied de la pierre dressée de Fatuahina. 23 - La première limite définie fut celle de Hoko. Elle partait de Mala'etoli, comprenait Mu'a jusqu'à Lanutavake et allait jusqu'à Ha'afuasia. 24 - Enfin, Fakate obtenait le territoire restant, Hihifo compris. » (Frimigacci et Pilioko, n.d. : 12)

La pierre de Fatuahina est le mémorial de cette partition qui est aussi l'ébauche d'une organisation territoriale à l'échelle de l'île. On peut y voir tant un réaménagement « administratif » et foncier impulsé par la «tutelle» tongienne que le règlement négocié entre "chefferies" locales pour un partage des accès à l'eau, à la forêt et aux meilleures terres de cultures.

Cet épisode qui fixe l'emprise territoriale des trois titres de chefferie semble correspondre en profondeur à une transformation des structures d'occupation, à un réaménagement foncier, sous l'effet de nouvelles formes de hiérarchies sociales, à une mise en place qui vise à une plus forte intégration politique.

Ces nouvelles délimitations qui partagent 'Uvea en trois districts, chacun sous le pouvoir éminent d'un titre de chefferie, se verront rapide- 


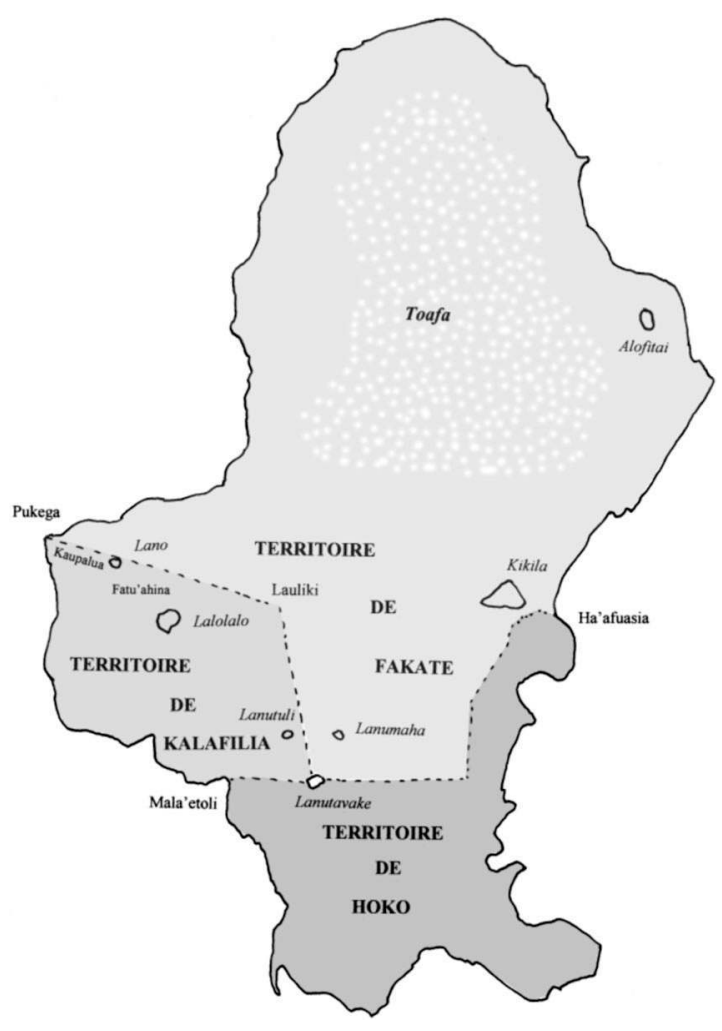

Figure 3. - Le partage territorial de 'Uvea.

ment contestées par Liaki, petit-fils de Fakate, et Huka, celui de Kalafilia, l'enjeu étant le lac de Lalolalo (cf. Corpus de traditions orales et Frimigacci et Pilioko, n.d. : 18-19). La tradition veut que ce différent ait été soumis à l'arbitrage du Tu'i Tonga ( $c f$. le 'eke [danse] de Vaimalau).

\section{- L'expédition de Kau'ulufonuafekai}

Le récit de l'expédition de Kau'ulufonuafekai à 'Uvea (cf. Corpus de traditions orales et Frimigacci et Pilioko, n.d. : pp. 25-29), motivée par la recherche de Malofaafa et Apitia, les assassins de sa mère, Tu'ivaekoloa ${ }^{34}$, est d'autant plus intéressant qu'on en connaît des variantes dans les traditions de Tonga, Niua et Futuna. Au-delà de l'événement qu'il rapporte ce récit a une valeur métaphorique. Ce pourrait être la synthèse de plusieurs expéditions tongiennes entreprises pour « remettre de l'ordre » dans une situation politique confuse, ce que laisse entendre la tradition de 'Uvea qui ajoute d'autres raisons à cette expédition.

L'analyse du récit permet de relier à travers les différents épisodes Ga'asialili à ceux que le père Joseph Henquel classait comme les «Anciens Rois » ('U Hau Afea). Sans parvenir à une chro- nologie généalogique précise, on peut néanmoins les resituer dans le schéma général. Havea Fakahau, Talapili, Talamohe et Fakahega se situeraient au même niveau généalogique, $17 \mathrm{ou}$ 16. G'a'asialili serait situé au même niveau (18) que Kau'ulufonuafekai voire au niveau inférieur, si l'on prend à la lettre la tradition tongienne qui en fait un «frère» [un germain] de Fakahega. Puakavahe et Toamisa se situeraient au niveau 16 et les principaux protagonistes du Lomipeau au niveau 15 sous le règne de Uluakimata dit Telea.

Cette tentative de reprendre en main les affaires sur l'île de 'Uvea se solda en définitive par un demi échec et le Tu'i Tonga dut renoncer à sa main-mise sur l'île :

« 67 - Le Tu'i Tonga ordonna aux deux assassins de rester à 'Uvea puis il déclara que 'Uvea n'aurait plus à s'acquitter du fatogia envers Tonga. Par contre il fit savoir que les Pelehake resteraient toujours sous la tutelle du Tu'i Tonga » (Frimigacci et Pilioko, n.d. : 28).

Il s'en suivit des relations d'un nouvel ordre avec Tonga marquées par le relâchement de la « tutelle » exercée et l'accélération du processusd'« assimilation » des lignées d'origines tongiennes.

\section{- La guerre dite du Molihina}

La guerre du Molihina, que Joseph Henquel situe au début de la dynastie des Takumasiva (sous Tuakalau ou Eiki), est sans doute légèrement antérieure. Elle aurait eu lieu à la fin de la période dominée par les rivalités et les alliances négociées entre les puissantes chefferies du sud de l'île fondées autour des titres de Hoko, Kalafilia, Fakate et des descendants des Ha'amea et Ha'avakatolo venus de Tonga. Une tentative de chronologie généalogique permet de la situer autour du niveau généalogique $15^{35}$, soit à la fin de la période des forts, qui voit les prémisses d'un nouvel ordre social visant à transcender les clivages territoriaux et les rapports de force.

Sous le commandement de Maufehi Huluava, les «chefferies» du nord (district actuel de Hihifo) se sont confrontées avec une coalition menée par le Tu'i 'Alegau, qui comprenait les trois titres de "chefferie » autochtones dominant la scène politique, Kalafilia, Fakate, Hoko assistés des Ha'amea et des Ha'avakatolo, les descendants des Tongiens.

Telle que nous la rapporte la tradition orale, la guerre du Molihina comporte plusieurs épisodes : le regroupement des forces du Maufehi sous 


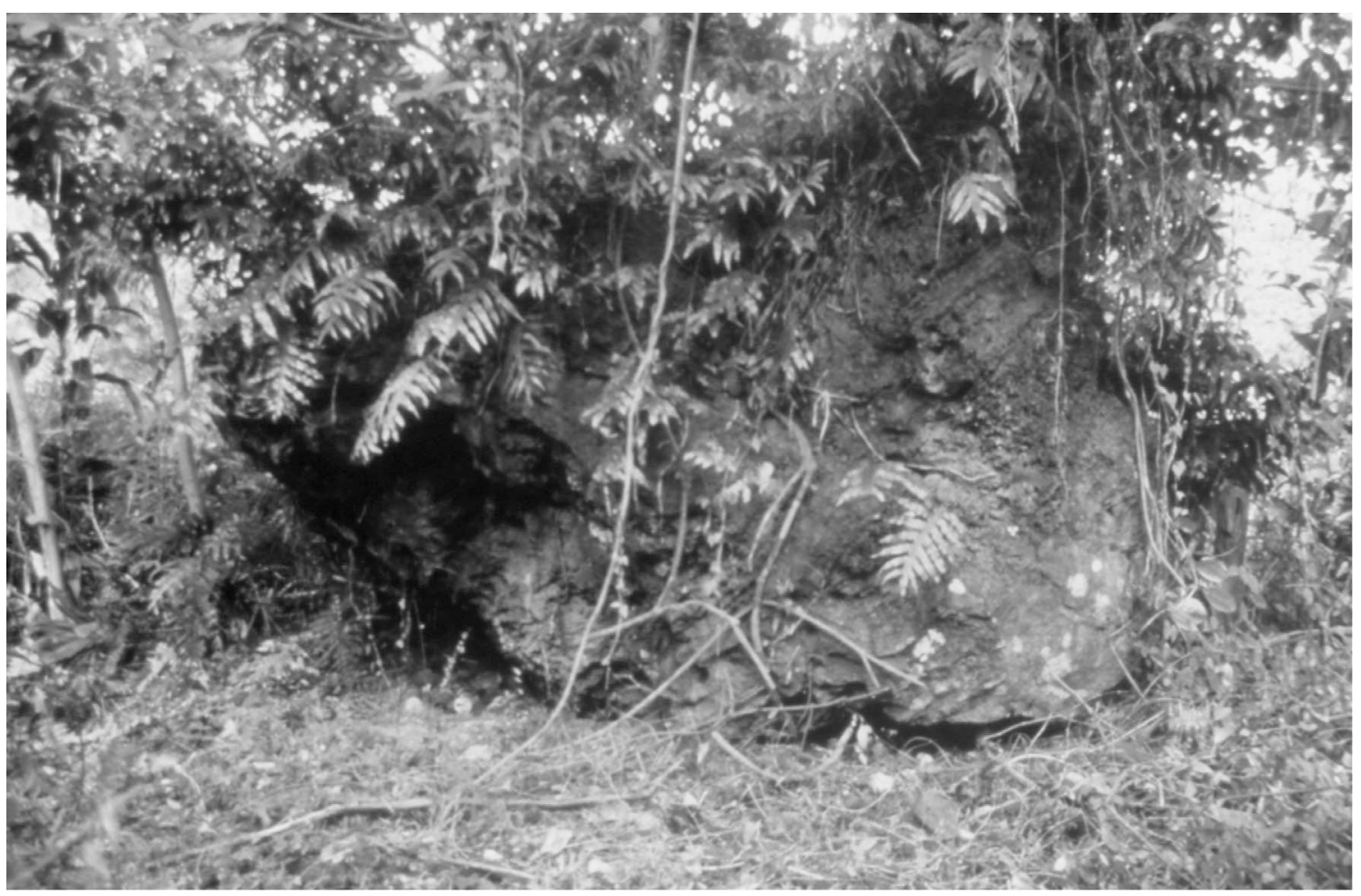

Рното 1. - La pierre de Fatuahina (MU 040) (cliché mission ORSTOM-CNRS)

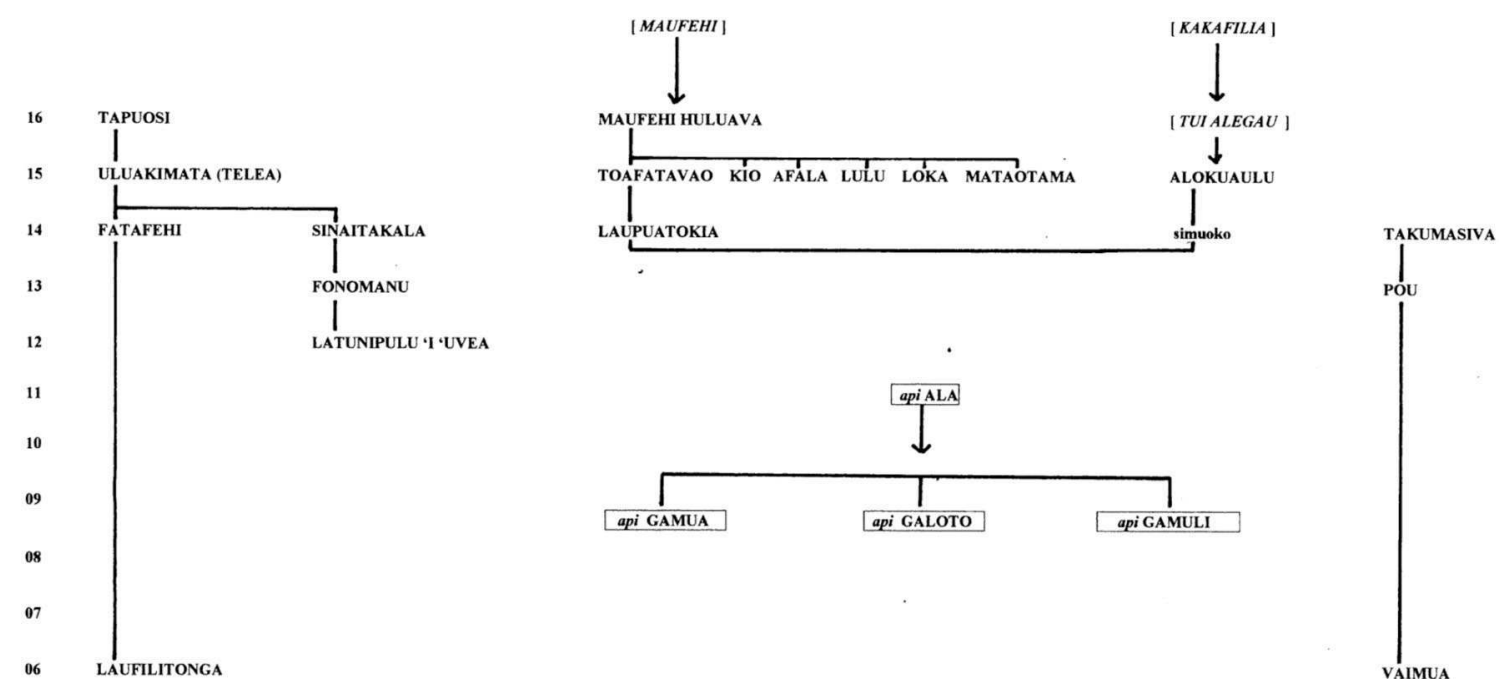

FIgURE 4. - Chronologie généalogique de la guerre du Molihina. 
le commandement de ses fils Toafatavao, Kio, Afala, Lulu, Loka et Mataotama; l'affrontement avec les forces de Fakate qui voit la victoire du Maufehi, dans la région de 'Utuloa sur la côte ouest; l'embarquement de Toafatavao et de ses guerriers pour Utuleve ; la confrontation décisive qui aurait eu lieu à To'ogatoto, à 'Utuleve et le massacre des forces du Maufehi ; l'extermination des gens d'Alele et «l'autorisation» de repeuplement de la région Nord. C'est le Tu'i 'Alegau, Laukuaulu qui présida au règlement définitif de la guerre du Molihina par le mariage de sa fille Simuoko avec Laupu'atokia, fils de Koli'ui, l'une des deux femmes de Toafatavao, fils de Maufehi Huluava, rescapée du massacre de la population d'Alele, après s'être réfugiée dans le toafa de Fuga'uvea.

Le règlement de la guerre du Molihina a provoqué un profond bouleversement des rapports de forces. Il signa le début d'un nouveau système d'intégration territoriale des " chefferies", de la domination du hau sur l'ensemble de l'île. Ce fut le début de l'ordre dynastique qui finit par s'imposer à l'île toute entière. Il faudra encore plusieurs générations et bien d'autres conflits entre les lignées dominantes pour aboutir à l'organisation du « royaume » de 'Uvea telle que nous la connaissons aujourd'hui. Elle eut aussi pour conséquence un réaménagement de l'emprise territoriale et foncière des titres de chefferie du Nord ${ }^{36}$. On peut penser que de cette époque $\left(12^{\mathrm{e}}\right.$ ou $13^{\mathrm{e}}$ niveau généalogique) date le partage de l'île en trois districts (Mua, Hahake, Hihifo) dans ses limites actuelles.

Si telle qu'elle est exprimée, la tradition de la guerre du Molihina se présente comme une succession d'événements qui traduisent sous une forme métaphorique une conjoncture réelle, il y a à l'évidence dans cette tradition plus que l'exaltation de certaines valeurs et la justification d'ordre symbolique de statuts, de configurations sociales et de rapports de force. La guerre du Molihina est un enchaînement d'événements présentant une certaine cohérence, une sorte de « micro-histoire » événementielle, un jeu de stratégies personnelles, reflet de provocations et d'affrontements, qui expriment les paramètres d'une période de tensions et de conflits, sur fond de "confrontations de prestige", dont il est impossible sans doute de préciser la chronologie en temps réel.

\section{- L'odyssée du Lomipeau}

Après la guerre du Molihina, l'odyssée du Lomipeau est l'épisode le plus emblématique des

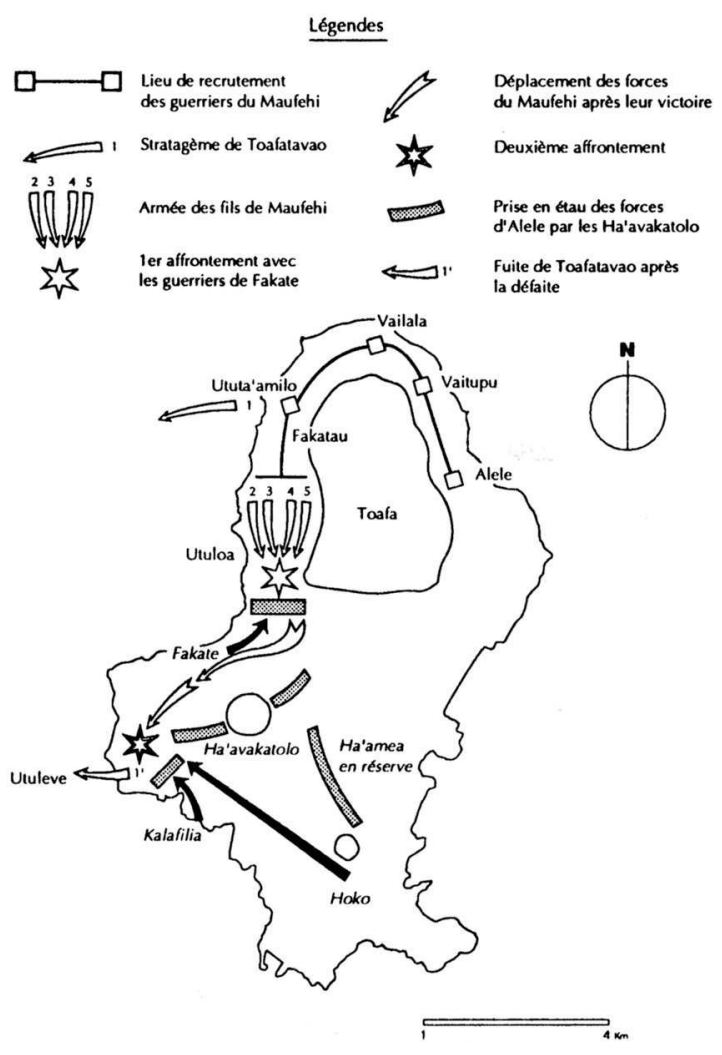

Figure 5. - La guerre du Molihina.

talanoa de 'Uvea, des traditions orales à visée historiographique. Mais contrairement aux deux épisodes précédents, c'est surtout sa portée archétypique et son architecture mythique et symbolique qui font tout l'intérêt de ce récit. Les références patronymiques sont hétérogènes et ne sont là, semble-t-il, que pour conforter la réalité de cette pirogue mythique, symbole de la " puissance » et du « savoir-faire » des gens de 'Uvea qui transcendent les contingences spatiotemporelles. C'est, avant tout, l'évocation d'un système d'échange et aussi d'un rapport de force en termes de prestige, entre Tonga et 'Uvea, d'ordre «économique » et "cérémoniel ». L'inhumation des Tu'i Tonga et des 'eiki requérait un appareillage des sépultures et s'accompagnait d'un cérémoniel d'autant plus élaboré que ces rituels funéraires présentent beaucoup des caractères qui ressortissent des «compétitions de prestige » qui, sous une forme ou une autre, sont au centre de la plus part des systèmes politiques océaniens et des préoccupations de ceux qui les incarnent, du « Bigman » au « Roi » en passant par le "Chef Suprême ». 'Uvea aurait « exporté » vers Tonga des matériaux - essentiel- 
lement des « dalles » de corail et de basalte ${ }^{37}-$, mais aussi des «spécialistes » et/ou des exécutants attachés à des 'aliki soucieux, avant tout, de faire valoir leur rang. Il faut rapprocher de ce contexte la " construction » à 'Uvea de sépultures (qui resteront parfois même inoccupées) par des grands 'eiki venus de Tonga qu'on évoque aussi à 'Uvea.

La géographie de la navigation du Lomipeau liée à des atterrages de prestige -, ainsi que le fait déjà noté par l'archéologue William McKern que les matériaux utilisés dans les monuments tongiens auxquels il est fait allusion ne proviendraient pas nécessairement de 'Uvea conforte ce jugement sur l'aspect archétypique du mythe (McKern, 1929).

Edwin W. Gifford (1924 : 67), en présentant la version tongienne du Lomipeau, précise qu'elle provient d'un informateur de Niua, originaire de 'Uvea, laissant entendre qu'elle n'aurait guère de contre-partie dans les traditions tongiennes. Par contre, dans sa thèse, 'Okusitino Mahina (1992) fait état, pour sa part, d'un corpus beaucoup plus riche de traditions se rapportant explicitement au Lomipeau et à ses atterrages. Cette version nous dit par contre beaucoup sur l'importance de la navigation et le statut des « chefs » de pirogue et des équipages. La maîtrise des relations maritimes avec Tonga aurait bien pu être l'un des enjeux stratégiques des rivalités politiques entre les « chefferies » du sud à la période des forts. C'est sans doute ainsi que l'on peut interpréter l'ancrage du mythe dans un référent patronymique qui renvoie à cette époque. Sur le plan plus "micro-événementiel », le mythe se réfère néanmoins à un épisode plus particulier qui aurait trait au règne de Telea à Tonga, dont on ne connaît pas tous les tenants et aboutissants, mais dont il a préservé la trace.

\section{Chronologie et périodisation: les apports de l'archéologie}

L'archéologie de 'Uvea nous a fourni les repères qui permettent de situer dans des intervalles en temps réel les périodes de l'histoire de l'île et la chronologie généalogique restituée à partir des traditions orales. Les recherches archéologiques, qui ont suivi la phase de l'inventaire, ont rendu possible la résolution d'un certain nombre de problèmes, notamment de situer la date des premiers peuplements, de périodiser son histoire, de mettre en évidence l'évolution des industries, tout particulièrement celle de la céramique et la date de son abandon (aux alentours de $\mathrm{XVI}^{\mathrm{e}}$ siècle). Les fouilles ont également permis de mieux cerner la période des constructions des grands monuments (vers l'an 1400). L'archéologie n'a malheureusement pas donné de réponses définitives, aux questions sur les origines de la domestication du cochon, de la poule et du chien, ni à celles des débuts de l'horticulture dans le contexte de 'Uvea.

La préhistoire de 'Uvea peut se diviser en quatre grandes périodes : la période Utuleve partagée en I et II -, la période Atuvalu, la période des forts et la période dynastique. Cette périodisation est présentée en détail ailleurs par Daniel Frimigacci (2000). Nous n'en reprendrons ici que le cadre et les principales conclusions sur des points essentiels à l'établissement $\mathrm{du}$ modèle diachronique qui sous-tend notre analyse de l'évolution des systèmes sociaux dans la perspective d'une anthropologie historique de la Polynésie occidentale. La figure 6 positionne cette périodisation dans la chronologie historique en temps réel.

\section{Utuleve : les découvreurs et les premiers occu- pants}

La période Utuleve débute autour de l'an 1000 $\mathrm{BC}^{38}$. Elle marque l'arrivée à 'Uvea de populations de langue austronésienne porteuses de la poterie Lapita. En fonction de l'évolution de la céramique, on peut partager la période Utuleve en deux périodes : Utuleve I (Lapita décoré) et Utuleve II (Lapita non décoré). Les premières installations (Utuleve I) sont essentiellement côtières et se limitent à la côte ouest, aux formations dunaires et marécageuses qui font face aux trois grandes passes du récif barrière : Avatolu, Fuga'uvea et Fatumanini. Tous les sites Lapita connus de la période Utuleve I correspondent à des implantations en bord de mer, sur des dunes et à proximité de zones marécageuses suivant un modèle très typique des campements des navigateurs Lapita découvreurs d'îles. La disparition de la céramique de type Utuleve I (décors Lapita) et la présence unique de la céramique de type Utuleve II (non décorée) signent les débuts de la colonisation de l'île par les descendants de ces mêmes découvreurs ayant pu, ou non, entraîner dans leur sillage d'autres composantes d'une population qui va progressivement occuper l'ensemble des zones côtières puis l'intérieur de

37. Une carrière d'extraction de ces dalles de basalte a été répertoriée à Ha'afuasia, Kasaua (HA061).

38. Pour la publication des datations relatives à cette période et ci-dessus (§ Datations archéologiques), voir Daniel Frimigacci (2000). Pour une discussion de ces datations controversées, voir Christophe Sand (2000) et plus avant (§ Datations archéologiques). 


\begin{tabular}{|c|c|}
\hline $\begin{array}{l}\text { Période Utuleve } \\
{[1000 \mathrm{BC}-1000 \mathrm{AD}]}\end{array}$ & $\begin{array}{l}\text { Présence de céramique de type Utuleve I et Utuleve II : } 1126 \text { BC } \pm 170 \text {. } \\
\text { (campements Lapita sites d'Utuleve et d'Utupoa). } \\
\text { Disparition de la céramique de type Utuleve I : } 200 \text { BC } \sim 100 \text { AD. }\end{array}$ \\
\hline $\begin{array}{l}\text { Période Atuvalu } \\
{[1000 \text { AD-1400 AD] }}\end{array}$ & $\begin{array}{l}\text { Disparition de la céramique de type Utuleve II : } 1285 \text { (site d'Utupoa). } \\
\text { Apparition de la céramique de type Utuleve III : 898/944 (site de Talietumu). } \\
\text { Sépulture de Puhi : } 1280 \pm 200 / 1390 \pm 100 \text { (site d'Atuvalu). } \\
\text { Monument antérieur à la résidence tongienne : } 1126 \pm 60 \text { (site deTalietumu). } \\
\text { Monument antérieur, sous le Malamatagata : } 1214 \pm 60 \text { (site d'Utuleve). }\end{array}$ \\
\hline $\begin{array}{l}\text { Période des forts } \\
{[1400 \text { AD-1460 AD] }}\end{array}$ & $\begin{array}{l}\text { Résidence du Talietumu : vers 1400 1450 (évaluation). } \\
\text { Règne de Kau'ulufonuafekai à Tonga : } \pm 1450 \sim 1470 \text { (estimation généalogi- } \\
\text { que). }\end{array}$ \\
\hline $\begin{array}{l}\text { Période dynastique } \\
\text { [1460 AD-Présent] }\end{array}$ & $\begin{array}{l}\text { Fin de la céramique Utuleve III : } 1528 \text { / } 1632 \text { (site Site d'Utupoa). } \\
\text { Règne de Takumasiva } \pm 1560 \sim 1580 \text { (estimation généalogique). } \\
\text { Découverte de 'Uvea par Samuel Wallis : } 1767 . \\
\text { Visite du capitaine J. Cook sous le règne de Paulaho à Tonga : } 2 \text { octobre } 1773 \text {. } \\
\text { Naissance de Vaimua (Soane Patita Lavelua) : } 1798 \sim 1799 . \\
\text { Visite du capitaine Moane sous le règne de Toifale à 'Uvea : } 1825 . \\
\text { Mort de Toifale : } 1829 . \\
\text { Pillage du Zebra, un sloop de guerre anglais : mai } 1832 . \\
\text { Arrivée des Maristes à 'Uvea : } 1 \text { novembre1837 AD. } \\
\text { Demande de protectorat français par S. P. Lavelua : } 1842 \text {, ratifié en } 1887 . \\
\text { Décés de Laufilitonga à Tonga : } 1865 \text {. }\end{array}$ \\
\hline
\end{tabular}

FIGURE 6. - Repères chronologiques.

l'île. Avec l'occupation de toute la zone côtière du nord au sud de la côte ouest, puis celle du sud, de l'est et des régions intérieures de l'île (Utuleve III) se met en place une horticulture qui reste dans un premier temps associée à l'exploitation du biotope marin.

\section{Atuvalu: le mythe de la grande chefferie}

La période Atuvalu commence avec l'apparition de la poterie de type Utuleve III, présente en quantité notable sur l'ensemble de l'île et des îlots, à l'exclusion toutefois du toafa. La répartition de cette poterie en surface traduit une démographie importante et une dispersion de l'habitat en petits «villages », antérieure aux regroupements qui vont s'opérer ultérieurement et à son redéploiement à la période dynastique. Elle s'achève avec Puhi et le partage territorial de 'Uvea. Le fort d'Atuvalu (MU020) construit au sommet de la pointe de Lausikula domine la mer d'une centaine de mètres, au lieu dit Atuvalu, et abrite la sépulture de Puhi. D'autres grands guerriers de 'Uvea y seraient également enterrés. D'après certaines traditions, bien après l'inhumation de Puhi, cet emplacement aurait été le lieu privilégié d'inhumation des premiers « hau » de 'Uvea et d'autres grands personnages - peutêtre même tongiens - dont la mémoire n'a pas toujours été préservée. Havea Fakahau y serait lui aussi enterré ainsi que les héros tongiens morts au cours de la guerre du Molihina. D'autres sépultures de la pointe de Lausikula se rapporteraient, plus généralement, à des chefs tongiens, notamment du lignage Ha'avakatolo qui occupaient cette partie de 'Uvea. Ohopulu, fille du premier Kalafilia y serait également enterrée. Les datations obtenues sur les os du squelette attribué à Puhi en font un site contemporain de la construction des premiers monuments retrouvés sous le Talietumu et le Malamatagata. Sa sépulture est de facture ancienne (type D) et le fort d'Atuvalu aurait été construit autour de la sépulture de Puhi beaucoup plus tard.

La période des forts : une implantation tongienne symbole de puissance sur fond de conflits intertribaux

Elle débute avec l'apparition des structures fortifiées que la tradition orale attribue aux Tongiens. Beaucoup d'indices et de traces d'ouvrages difficilement identifiables laissent à penser que les constructions en pierre, peut-être à d'autres fins que résidentielles ou défensives, sont une pratique antérieure à l'arrivée des Tongiens avec Ga'asialili. Le fort de Ha'afuasia (HA058) en référence à Tauloko, pourrait être la première résidence fortifiée connue. C'est un fort en terre, construit sur le 'api Havaiki, entouré d'un fossé défensif à l'ouest, protégé par la falaise du bord de mer à l'est. D'autres forts en terre, comme ceux de Matagaika (MU126) et de Fugakolo (MU095) semblent contemporains du fort de Ha'afuasia. 


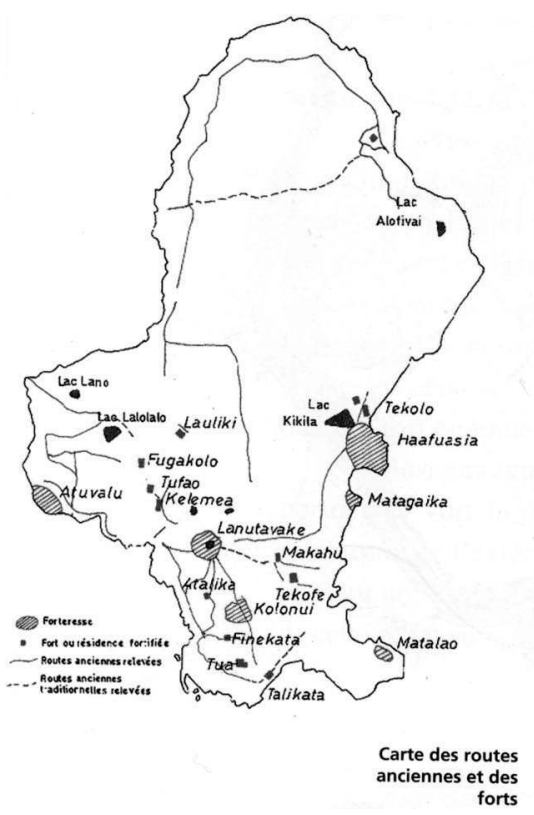

Figure 7. - Forts et routes anciennes.

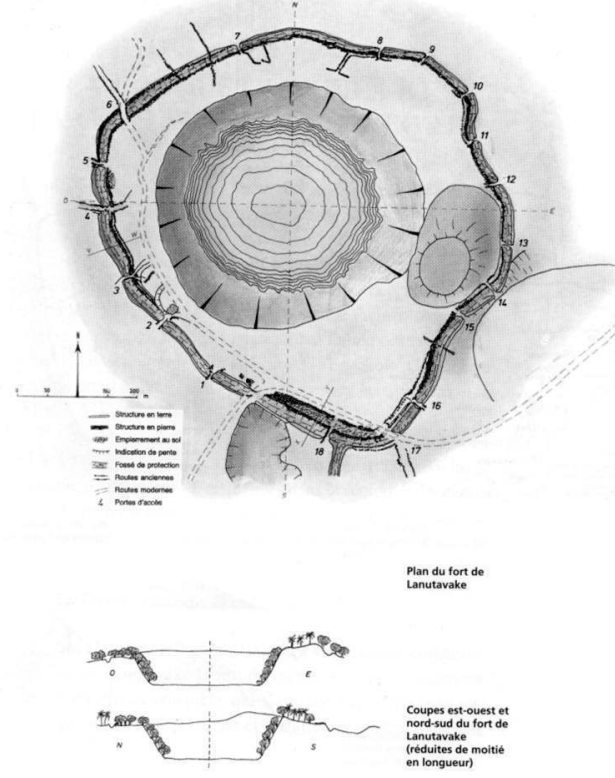

Figure 8. - Le fort de Lanutavake.

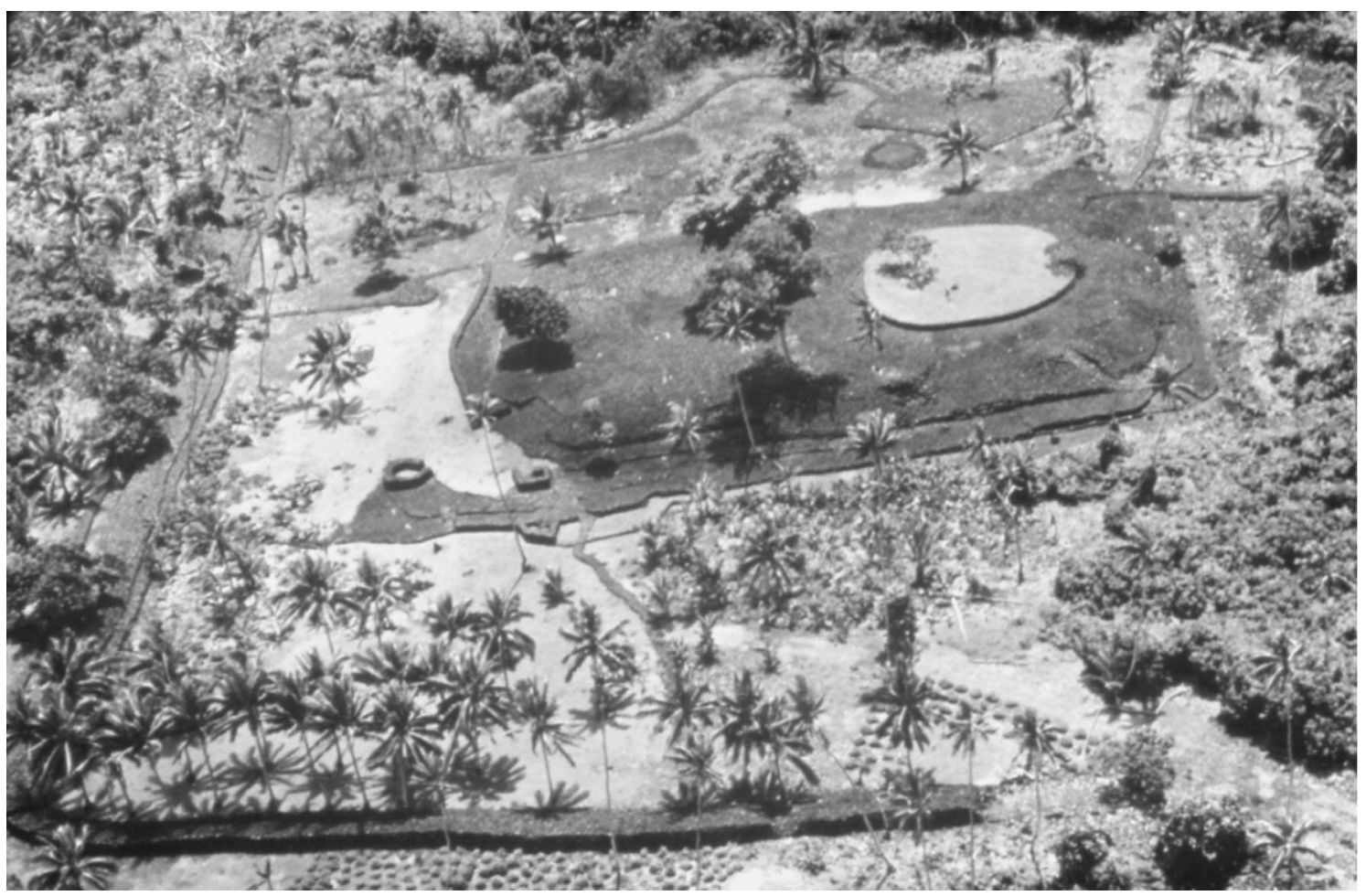

Рното 2. - La résidence du Talietumu (MU029) (cliché : mission ORSTOM-CNRS). 
Ultérieurement, les chefs tongiens des lignages Ha'amea et Ha'avakatolo arrivent et font construire une série de forts et de résidences fortifiées dans la région de Mu'a autour du lac Lanutavake ( $c f$. Fig. 8), qu'ils entourent d'un dispositif défensif. Cet ouvrage (MU060), pièce mâ̂tresse de leur dispositif, est au point de jonction des trois territoires dévolus à Hoko, Fakate et Kalafilia.

"Possibly Hoko, Kalafilia and Fakate were chiefs of a pre-Tongan population. » (Burrows, $1937: 171)$

Un ensemble de routes fortifiées construites sur le modèle tongien (McKern, 1929) rayonnent à partir des dix-huit portes de ce fort, permettant de rejoindre les différentes places fortes et autres résidences du dispositif d'ensemble mis en place par les Tongiens. L'intérieur du fort de Lanutavake comprenait l'eau du lac mais aussi des terres de culture. Il était entouré de fossés défensifs et de murs en pierre sèche munis de postes de guets élaborés (Frimigacci et Hardy 1997).

\section{La période dynastique :}

la stabilité institutionnelle

La stabilité des institutions se produit sur fond de luttes intestines. Dans une première phase, le titre de Hoko d'abord, puis celui de Mahe, chargés d'introniser cérémoniellement les premiers hau et de garantir ainsi leur légitimité, semblent avoir joué, en quelque sorte, un rôle d'arbitrage. Le kau 'aliki, le conseil qui entoure aujourd'hui le $h a u$, va progressivement s'institutionnaliser, s'élargir et jouer un rôle déterminant pour réguler et stabiliser le système politique. Le réseau des alliances matrimoniales redoublées entre lignées dominantes et entre les titres de chefferie aura un impact similaire.

Elle débute avec l'avènement de Takumasiva, le fondateur de l'actuelle dynastie, brièvement interrompue par une tentative d'usurpation du hau, par Tuakalau et Eiki, deux chefs d'origine tongienne, puis celui des Vehi'ika peu de temps après. De Takumasiva on sait seulement qu'il prend la place de Siulano. Il pourrait être lié à Ha'afuasia et au titre de Hoko. Vehi'ika, lui, viendrait de Finekata et serait plus directement lié au titre de Kalafilia. Ces deux lignes dynastiques, fondatrices de la «monarchie » de 'Uvea, vont progressivement se fondre l'une dans l'autre au fil des générations par le biais de mariages répétés, au bénéfice des Takumasiva. L'interrègne de Kulitea et de ses petits-fils Lavekava et
Hiva montre la fragilité de cette architecture parentale et la part d'aléa que le jeu des rapports de force extérieurs introduit en pratique dans la fonctionnalité d'un système construit idéalement, et en théorie, sur le rang, la filiation patrilinéaire et l'aînesse. On notera ici encore comment des mariages ultérieurs, notamment en renforçant ou en réaménageant l'image d'une «filiation parentale» entre les porteurs successif $\mathrm{du} h a u$, s'efforceront de constituer les fondements d'une sorte de légitimation a posteriori de ce qui autrement n'aurait pas manquer d'apparaître, à un titre ou un autre, comme une usurpation, comme un précédent. Dans cette logique, la référence permanente à la "personne » du hau comme l'incarnation des valeurs de la société uvéenne justifie cette part de choix optionnel qui contrebalance la filiation et semble à bien des égards constitutif de la structure même de cette organisation politique.

\section{La formation et l'évolution du royaume de 'Uvea}

Quel schéma plausible de reconstruction de l'histoire pré-européenne de 'Uvea peut-on alors suggérer qui soit en cohérence avec les données connues de l'histoire régionale ? Que peut-on en inférer quand à la structure sociale de cette « royauté » polynésienne?

On sait que 'Uvea a été découverte et peuplée par des navigateurs Lapita qui y ont développé au moins trois campements : Utuleve, Fatufoa et 'Utupoa, tous situés sur la côte ouest, aujourd'hui inhabitée, sous le vent de l'île. Ces campements de faible étendue sont situés dans des environnements écologiques très similaires et typiques du contexte environnemental des sites Lapita de la Nouvelle-Calédonie, du Vanuatu, de Fidji et de la Polynésie occidentale, sur des dunes, en face des passes du récif barrière, à proximité de zones marécageuses (Frimigacci, 1980). Ils sont associés à la poterie de type Utuleve I (Lapita décoré à dégraissant corallien). Les fouilles des deux sites d'Utuleve et de 'Utupoa ont fourni des stratigraphies et des datations concordantes. Le site de Fatufoa, détruit par des extractions de sable, n'a pas permis de retrouver une stratigraphie fiable. Les datations obtenues, qui s'échelonnent de $2986 \pm$ $70 \mathrm{BP}$ à $2686 \pm 170 \mathrm{BP}$ pour les niveaux de la période Utuleve I, sont congruentes avec celles obtenues pour Fidji, Tonga, Samoa ${ }^{39}$ et suggè-

39. Nous sommes conscients que les datations déjà anciennes concernant les chronologies de Fidji et Samoa sur lesquelles on avait l'habitude de s'appuyer pour les synthèses régionales (Kirch, 1997 ; Spriggs, 1997) sont aujourd'hui sérieusement discutées et revues à la baisse : le peuplement de ces archipels pourrait être plus «récent » qu'on ne l'avait 
rent quelques remarques sur la dynamique du peuplement.

Le peuplement de 'Uvea est contemporain de celui des îles et archipels voisins comme si la découverte et la première colonisation de cet ensemble régional s'étaient effectuées dans une même dynamique de peuplement mais peut-être selon deux routes de navigation divergentes: Fidji, Tonga d'une part, Futuna, 'Uvea, Samoa, d'autre part. C'est là une hypothèse suggérée par la classification interne des langues polynésiennes, mais aussi par d'autres facteurs ${ }^{40}$. La très grande proximité chronologique des datations obtenues pour les sites Lapita des Santa Cruz et $\mathrm{du}$ Nord Vanuatu suggère une certaine continuité relationnelle et culturelle le long de l'axe général de peuplement, donc une première colonisation peut-être antérieure à la différenciation culturelle Mélanésie / Polynésie. Par contre la distribution de l'obsidienne et des verres volcaniques plaide pour une " rupture " très rapide des communications en retour le long de l'axe du peuplement, peu de temps après la découverte des îles, ce que renforce l'image d'une plus grande continuité d'occupation dans la stratigraphie des sites, comparée aux Santa Cruz (Nendö, Reef islands) et au nord Vanuatu (Aore, Malo) (Green, 1976 ; McKoy et Cleghorn, 1998 ; Hedrick, 1971 et n.d. ; Galipaud, 2000 ; Pineda et Galipaud, 1998 ; Bedford, 2003).

La continuité d'occupation des sites visualisée dans les stratigraphies et la présence concomitante, pendant quelque huit cents ans, de la poterie décorée de type Utuleve I et de la poterie plus « utilitaire » de type Utuleve II dans les niveaux anciens, viennent renforcer l'idée que ces campements dunaires n'étaient pas saisonniers comme cela pourrait être le cas, dans un premier temps du moins, pour d'autres sites Lapita des Santa Cruz ou du Nord Vanuatu (communication personnelle de Jean-Christophe Galipaud) ${ }^{41}$ mais qu'ils constituèrent d'emblée des sites d'occupation plus permanents. On observe très rapidement une extension du site d'occupation vers l'intérieur des terres, associée exclusivement à la poterie de type Utuleve II et ensuite Utuleve III qui renforce ce sentiment et suggère une transition rapide entre une adaptation centrée sur l'exploitation du biotope marin et une horticulture de subsistance dominante. Cette observa- tion renvoie à une problématique plus générale. Convient-il ou non de distinguer, pour comprendre le processus de peuplement de ces îles de «l'Océanie lointaine », les navigateurs - découvreurs Lapita associés à la poterie décorée dont la fonction pourrait relever des « objets de prestiges ", des colonisateurs Lapita associés à un type ultérieur de poterie non décorée plus fonctionnelle (plainware) ? Doit-on alors les considérer, d'un point de vue sociologique, comme une composante d'un système social plus complexe, une sorte "d'aristocratie » spécialisée dans la navigation et la découverte ou comme une population de navigateurs avec un mode de vie et une adaptation très spécifiques entraînant dans son sillage d'autres composantes de la population des premiers colons responsables de la pénétration des îles et du développement de l'horticulture?

À la poterie de type Utuleve II correspond l'extension des occupations à pratiquement toute la côte ouest de l'île (une quinzaine de sites inventoriés répartis du Nord au Sud), une extension côtière qui va de pair avec les débuts d'une colonisation de l'intérieur de l'île. Avec l'apparition de la poterie de type Utuleve III débute la période Atuvalu. La densité et la répartition de cette céramique présente partout dans l'île, y compris sur les îlots du lagon, suggèrent une forte croissance de la démographie et une dispersion de l'habitat sur l'ensemble de l'île sous forme de petites communautés résidentielles installées près ou au sein même de leurs terres de culture. Ce modèle d'occupation pourrait sousentendre des relations plutôt pacifiques entre ces communautés.

L'émergence, dans ce contexte, d'un système de « chefferies à titres", en partie enraciné dans la tradition mythique, reste la partie la plus obscure de la proto-histoire de 'Uvea, la plus sujette à spéculation. À défaut d'un modèle avéré, on peut avancer deux hypothèses, qui d'ailleurs ne s'excluent pas nécessairement. Soit les premiers colonisateurs seraient venus porteurs d'un système social déjà très hiérarchisé, c'est l'idée d'une société proto-polynésienne constituée, déjà différenciée culturellement avant même le peuplement de ces îles, soit un système d'autorité hiérarchisée se serait développé dans un contexte sociologique, si ce n'est d'environnement, en

laissé entendre. Notre proposition reste suffisamment générale pour qu'il ne soit pas indispensable d'entrer dans ce débat.

40. Pour une présentation synthétique plus « exhaustive » de l'apport des linguistes à cette problématique (classification des langues et reconstruction linguistique du proto-polynésien), voir Kirch (2001).

41. L'interprétation plus avant de cette association dans les mêmes niveaux de la poterie « Lapita décorée » avec du « Lapita non décoré » - et/ou un autre type de céramique - renvoie à une problématique régionale, trop complexe pour être abordée ici. 
tout point similaire à ceux que l'on peut décrire pour la Mélanésie, développant en les articulant d'une façon propre les mêmes valeurs de hiérarchie, de rang et de compétition communes à tous les systèmes sociopolitiques océaniens, sous la pression de contraintes spécifiques et dans un contexte historique particulier. Cette seconde hypothèse conduit à devoir repenser autrement l'identification et la diachronie de la coupure culturelle Mélanésie/Polynésie. La pauvreté relative du milieu en ressources naturelles, une forte démographie, la maîtrise de la navigation hauturière avec l'impact précoce et les interactions d'un modèle d'organisation sociale hautement diversifié sont, dans le cas de 'Uvea, parmi les facteurs à l'œuvre. Nous restons ici très dépendants d'une meilleure compréhension de la divergence dans les processus historiques de formation de la royauté tongienne d'une part, du système de titres (matai) samoan et des diverses «chefferies» (matanitu) et confédérations de «chefferies» fidjiennes, d'autre part, sans oublier l'éclairage que peut apporter la « dynamique » historique des " chefferies à titres » du Centre et Sud Vanuatu.

La sépulture de Puhi situe la fin de cette " période Atuvalu » qui a vu la formation et/ou la consolidation de "chefferies autonomes" locales dans le sud de l'île mais sans doute aussi dans le nord (Hihifo) et le long de la côte ouest. C'est ce que suggère certaines des traditions que nous avons recueillies se rapportant aux titres de chefferie: Heu, Maufehi, 'Utumaka ou aux anciennes implantations Faka'utu, Fatai et 'Ahoa et aux titres qui pourraient leur être associés. Les mythes d'origine des titres de Hoko, Kalafilia, Fakate mais aussi de Heu et Maufehi s'enracinent dans cette période Atuvalu à laquelle ils se rattachent implicitement, voire même y font directement référence dans certaines versions, par ailleurs les plus controversées.

Puhi, mais surtout l'ensemble funéraire d'Atuvalu, les « huit alignés », possèdent dans la tradition orale de 'Uvea une valeur symbolique forte. Atuvalu qui réunirait dans un même lieu les premiers hau, plus ou moins mythique, de 'Uvea - et tous ceux que l'île pouvait compter comme héros prestigieux - est un lieu fondateur de «l'autochtonie culturelle». Cette sépulture collective est la représentation en grande partie idéologique de l'idéal d'une " grande chefferie » unitaire, probablement mythique, mais en train de se mettre en place dans les faits. Cette période de l'histoire s'achève enfin sur le partage de 'Uvea entre les trois titres de chefferie: Hoko, Kalafilia, Fakate.
Dans ce contexte, Kau'ulufonuafekai, poursuivant l'œuvre entreprise par son père Takalaua, tente de prendre pied à 'Uvea, d'y implanter une colonie tongienne et d'intégrer définitivement l'île dans l'aire d'influence tongienne, dans son système territorial et sous son autorité. 'Uvea se verrait donc soumis au fatogia, au tribut annuel que le Tu'i Ha'atakaluaua aura la charge de faire collecter pour le Tu'i Tonga. Pour réaliser ce projet, Kau'ulufonuafekai envoie Ga'asialili qui va s'appuyer sur deux grands lignages, les Ha'amea et les Ha'avakatolo, qui jouent un rôle déterminant dans la réorganisation et l'expansion territoriale de la royauté tongienne puis vont prendre une place toute aussi importante dans l'histoire de 'Uvea.

Il semble que les choses ne se soient pas passées aussi facilement que prévu. Les Tongiens s'implanteront au point de convergence des limites territoriales des trois chefferies autochtones sous le leadership respectif de Hoko, Kalafilia et Fakate. Les Ha'amea s'installèrent à Muli'utu, les Ha'avakatolo à Tapuhia. Puis, à partir du fort de Lanutavake, les Ha'amea s'étendront vers le sud et construiront le fort de Atalika, les Ha'avakatolo vers l'est, vers Finekata, où ils construiront les résidences du Talietumu et Meito. Le fort de Fugakolo faisait aussi faire partie de ce dispositif. Cette implantation semble s'être faite au détriment de Fakate sur un plan territorial. La tradition orale suggère que les Tongiens se soient appuyés sur le titre de Hoko - ou allié avec lui pour s'installer au centre du système territorial des « chefferies à titres » autochtones et étendre leur aire d'influence ${ }^{42}$. Par analogie avec ce que l'on connaît ailleurs en Océanie, Hoko jouerait un rôle similaire à celui joué à Fidji ou en Nouvelle-Calédonie par les "maîtres du sol», remettant la "chefferie » dans les mains des " étrangers ", de ceux venus d'ailleurs (Guiart, 1963 ; Bensa, 2000; Sahlins, 1981a et 1985). C'est semble-t-il plutôt dans ce sens que comme la résultante d'une " conquête coloniale » qu'il convient d'interpréter le fait que :

« 28 - Tauloko, premier roi de 'Uvea, fut intronisé par Hoko à l'aube du peuplement de 'Uvea. » (Frimigacci et Pilioko, n.d. : 14)

Des tensions et des luttes d'influence seraient apparues ultérieurement entre les Ha'amea et les Ha'avakatolo, sur fond d'alliances et de renversements d'alliances entre lignées locales et lignées d'origine tongienne, ces dernières ayant tendance à progressivement fusionner avec les populations locales. 


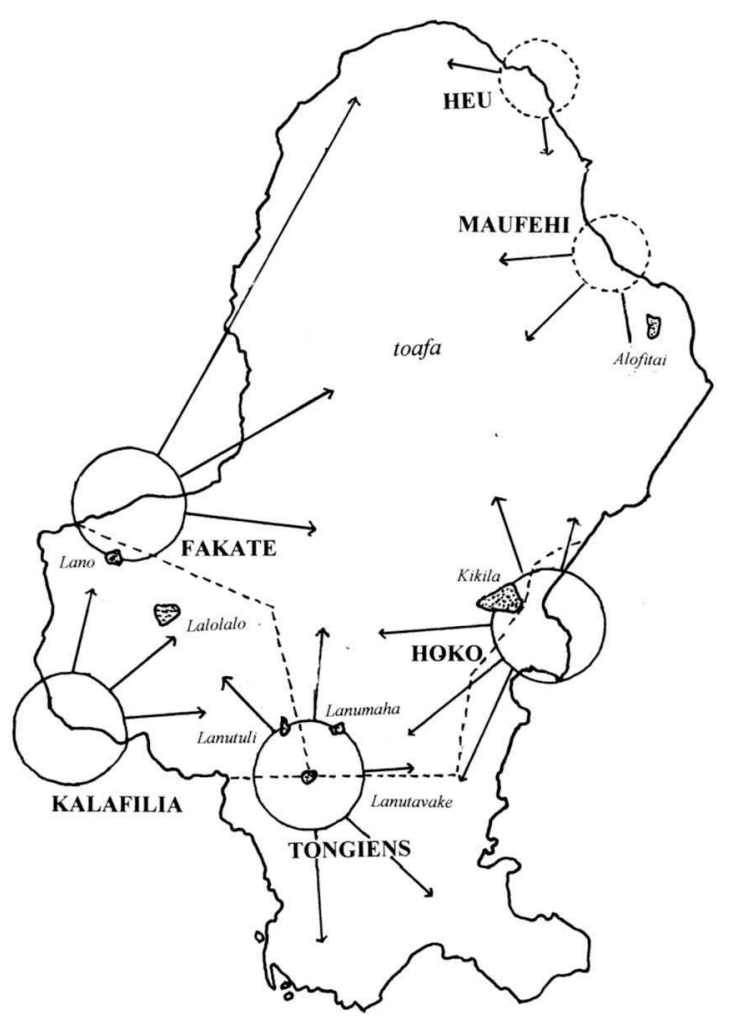

FIGURE 9. - Les chefferies de 'Uvea et leurs aires d'influence à la période pré-dynastique.

Il est difficile de se faire une idée plus précise en terme d'allégeance formelle, de rang ou de hiérarchie, des rapports entre ces différents titres de « chefferie » qui semblent le plus souvent placés par la tradition orale sur le même plan. L'image des rapports de force que l'on peut en retirer suggère une large autonomie des « chefferies » et une organisation fluctuante des alliances autour du titre de Hoko, d'un côté, du titre de Kalafilia (Tu'i Alegau) de l'autre.

« Altogether, it seems clear that in this early time the Tui Alagau was an independent ruler...In sum, much of the early history deals with fights among rival chiefs, and any assumption of united rule at that period is misleading. » (Burrows, 1937: 40 ; souligné par nous)

La formation sociale de 'Uvea nous apparaît à cette époque comme une société segmentée en plusieurs unités territoriales et de parenté 《 groupes tribaux » ou « lignagers »- largement autonomes sur un plan politique mais en conflit pour s'assurer une domination sur un ensemble territorial en formation. Étaient-elles liées les unes aux autres par un quelconque système d'échange et/ou de prestations cérémonielles, sous quelle forme et avec quelles incidences, c'est plus que vraisemblable, bien qu'il soit difficile aujourd'hui d'en décider. Le texte du Talanoa $k i$
'Uvea nei évoque bien un contexte de compétition de prestige mais ne fournit que très peu d'informations sur sa raison d'être et son éventuel organisation. La guerre du Molihina signe la fin de cette période et la mise en place d'une autre forme plus spécifique d'organisation sociopolitique : le « royaume» de 'Uvea.

On voit disparaître tour à tour des titres de chefferie aussi important que celui du Tu'i'Alegau, du Tu'i Lauliki ou du Tu'i Ha'amea... L'importance politique et le rôle joué par le Hoko, le Kalafilia et le Fakate vont aussi progressivement diminuer au fur et à mesure que s'affirme l'organisation «monarchique», à l'instar du rang qu'ils ont pu occuper à l'origine dans la hiérarchie des titres. Cette perte d'influence s'est faite au bénéfice des titres qui vont bientôt fournir l'architecture du kau aliki, la structure de l'exécutif du hau de 'Uvea. Dans l'ordre d'antériorité suggéré par la tradition orale, le Mahe, un titre qui apparaît dès la période des forts au niveau généalogique 13, va jouer un rôle pivot dans la mise en place du modèle dynastique. C'est sous le règne des descendants de Vehi'ika que l'on voit apparaître les ancêtres éponymes des principaux titres du kau 'aliki et de chefferies de village n'ayant pas " hérité » d'un titre antérieur ( $c f$. Fig. 10).

C'est aussi à cette période, ou peut-être un peu avant, que se met progressivement en place un nouveau modèle d'organisation de l'espace et de gestion des ressources naturelles, qu'on pourrait dire en "tranches de gâteau » (Vienne, 1998), typique de cette région de la Polynésie, puisque Futuna et Samoa en offrent deux variantes, qui s'appuie sur une redistribution linéaire de l'habitat. Chacun préserve - à l'échelle du 'apilkaiga un égal accès aux ressources naturelles indispensables (platier récifal, terrasses d'habitation, terres de cultures...) et les espaces de collecte comme le toafa, la réserve forestière (vao tapu) et, dans une certaine mesure, la mise à disposition des zones de pêche et des plots de culture (fuhi) dans les tarodières irriguées, passent sous le contrôle et la gestion directe du kau' 'aliki et des titres de chefferie (Vienne, 1998 et 1999). Le système dynastique va se développer et se structurer autour de plusieurs dynamiques, produits des stratégies de groupes territoriaux et de lignées en compétition sous la conduite de leaders " guerriers », de 'aliki toa.

Voyons maintenant les intermariages entre les descendants des Takumasiva et ceux de Vehi'ika - sans oublier ceux conclus par la suite avec les descendants du Kulitea - et entre les lignées collatérales de chacun de ces groupes. L'intégration par le biais des mariages contribue, sur le 


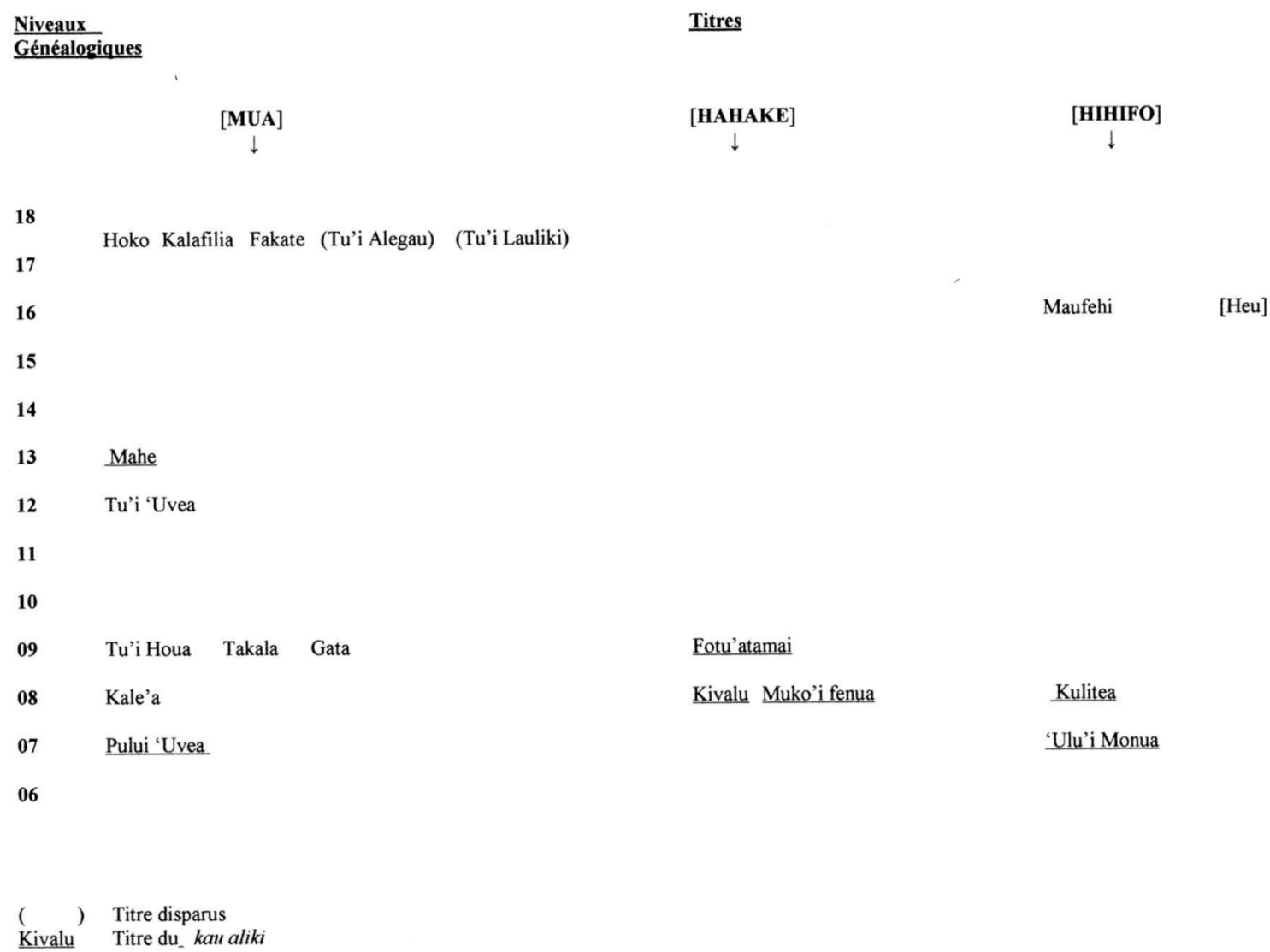

Figure 10. - Position chronologique de l'apparition des titres de chefferie dans l'histoire de 'Uvea.

plan de la structure, à dépasser les segmentations de groupes sociaux qui se définissent tout autant, si ce n'est plus, sur le plan territorial que sur celui de la parenté. C'est comme si on avait à faire à une structure ambivalente qui oscillerait entre deux polarités : générer des groupements territoriaux politiquement autonomes, organisés par la parenté ou intégrer ces groupes dans un ensemble plus large, détaché de son identité territoriale, construit aussi sur la parenté, mais visant à transcender les clivages de localité en terme de pouvoir, de rang et de hiérarchie. C'est ce « déracinement» du hau qui le "sacralise », qui permet d'imposer la fiction d'une « filiation dynastique »-dont nous avons souligné l'arbitraire, puisqu'elle procède ici d'une recomposition, souvent à posteriori, du tissu généalogique par le biais d'habiles stratégies matrimoniales - et instrumentalise l'autorité attachée, par idéal et consensus, à la fonction.

L'intégration des titres qui vont former le kau 'aliki dans l'architecture généalogique du hau, tend à formaliser la coupure 'alikiltua en terme d'opposition de statut et non plus en terme de "distance généalogique » ou de relation " d'aîné/cadet ». Elle permet de relayer, par les « fonctions » génériques - et symboliques - attachées à ces titres, la construction d'une rationalité idéologique autoritaire qui s'associe à l'exercice du pouvoir et le conforte.

«In old days the power of chiefs was so absolute that commoners were little better off than slaves. " (Burrows, $1937:$ 37)

Il en résulte une stratification sociale qui oppose une minorité de lignées apparentées formant une structure ramifiée, en partie hiérarchisée, centrée autour de la détention et de la transmission du hau, à un réseau de groupes locaux de parenté qui, à l'inverse, tendent à reproduire la structure lignagère segmentaire dont ils sont issus. La première monopolise l'autorité légitime et l'essentiel du pouvoir politique, à l'encontre de celle qu'exercent de fait, à l'échelle locale et parentale, le chef de la communauté villageoise, pule kolo, et les " aînés 》 des groupes résidentiels de parenté, pule kaiga (matua kaiga), au sein du fono fenua, le conseil de village. La «soumission » des titres de chefferie de village, pule kolo, au hau, par l'intermédiaire du kau 'aliki ( $k a u$ fa' $u$ ) et de ses exécutants réalise l'intégration structurale de cette formation sociale. La notion de kau'aliki renvoie aux notions de rang, de hiérarchie, de fonction rituelle au sens d'Edmund 
Leach (1954 : 10-14), celle de kau fa'u à l'exercice du pouvoir exécutif imparti à cette hiérarchie. On ne peut s'empêcher de noter, à titre comparatif, combien ce processus d'intégration politique qui résulte de l'interaction entre deux structures offre de similitudes formelles avec celui qui présida à la formation et à l'organisation du muang (principautés) thaï en Asie du Sud-Est (Condominas, 1976 ; Lemoine, 1997 ; Vienne et Goudineau, 2001). C'est aussi par rapport à cette dialectique des structures qu'il faudrait comprendre le «dualisme» du hau qu'on observe de façon récurrente dans l'histoire de 'Uvea. C'est aussi en tenant compte d'une telle perspective qu'il faudrait aborder le problème de la nature du kaiga qui fait toujours débat.

La coupure généalogique qui s'instaure en terme de stratification sociale entre le $k a u$ 'aliki et les titres de chefferie villageoise, dont beaucoup sont pourtant, à l'origine, généalogiquement liés au hau, conduit à consolider l'intégration territoriale - et politique - par l'émergence d'une strate "administrative» d'exécutants (matapule, mu'a, faipule, etc.) à laquelle est déléguée une partie du pouvoir d'exécution du kau $f a^{\prime} u$. Cette segmentation ne procède pas de la seule logique du développement des structures. Elle résulte plutôt d'un processus extrêmement complexe dans sa dynamique même, où les rapports de force se construisent autour de stratégies qui s'efforcent tour à tour de légitimer ou de contester l'ordre établi hérité en se référant à une symbolique des valeurs, à une forme d'institutionnalisation idéologique des conflits autour d'une certaine image du chef, du hau. Le hau est plus conceptualisé comme un être exemplaire, admiré et respecté, dont la conduite incarne un idéal de valeurs dont chacun se sent plus ou moins porteur à un titre ou à un autre, que comme un être sacralisé dans son statut, et d'une autre nature, qui en fait l'incarnation des dieux comme peut l'être le Tu'i Tonga à cette époque. Ce sont alors les motivations et les mobiles de ces stratégies, culturellement codifiées, qu'il conviendrait de mieux décrypter. On verra des tentatives de maintenir dans l'aire d'influence du kau 'aliki des titres de chefferies de villages ou à l'inverse de redonner, quand nécessaire, une assise territoriale de fait aux titres du kau 'aliki que la pensée politique définit essentiellement par leur fonction et leur rang cérémoniel. Les stratégies que nous venons d'évoquer s'enracinent, en outre, dans des dynamiques foncières auxquelles elles se trouvent confrontées, ce qui a aussi des effets primordiaux sur la structure politique.

\section{En guise de conclusion}

Le schéma avancé s'appuie sur une analyse plus en terme de compréhension - au sens de Max Weber - que sur une interprétation étroitement empirique des données factuelles. En ce sens, il reste nécessairement entaché d'imprécisions et sujet à débat. Nous avons surtout tenté de mettre en lumière une cohérence sociologique sous-jacente qui donne un sens aux événements probables mémorisés et rapportés par la tradition orale à visée historiographique, en nous gardant de trop de romantisme et d'ethnocentrisme conceptuel ${ }^{43}$.

Les progrès que l'on peut attendre des développements futurs de l'anthropologie historique en Océanie ${ }^{44}$ qui a pris un nouvel essor à l'aube des années 1980, grâce en particulier aux avancées considérables de la recherche archéologique et linguistique ainsi qu'au renouvellement des problématiques anthropologiques qui lui sont associées, permettront d'affiner à l'avenir ce schéma de reconstruction de l'histoire préeuropéenne de 'Uvea. Le recours toujours possible à de nouvelles sources d'informations sur l'histoire à tradition orale de cette région du Pacifique et une relecture critique des sources anciennes devraient permettre d'encore mieux cerner l'insertion dans le temps réel historique de cette diachronie, tout comme une mise en perspective ethnohistorique comparative des processus d'évolution et/ou de transformation des sociétés insulaires océaniennes dans le long terme nous permet d'en mieux comprendre la nature même. Elle jette un nouvel éclairage sur les formes d'organisation sociale, les relations d'échanges, la nature du pouvoir, la permanence d'une dialectique des structures qui se profile derrière le décryptage des conjonctures historiques, de l'événementiel, dans l'ordre du mythe ou de la culture ${ }^{45}$.

Si à première vue, la royauté de 'Uvea semblait un décalque des institutions tongiennes, on voit qu'elle en est profondément distincte d'un point

43. Ceux-là même que dénonce Jean Guiart (2005) à propos de la présentation par Christophe Sand de la préhistoire de 'Uvea et de Futuna dans le livre de Serge Dunis (2004).

44. Au sens où l'entend Patrick Kirch (2001). Voir aussi Elisabeth Bott (1982), Antony Hooper et Judith Huntsman (1985), Alan Howard et Robert Borovsky (1989), Valerio Valeri (1985), Marshall Sahlins (1981b et 1985), etc.

45. Sur la façon d'appréhender « l'événement » dans ses rapports avec la « structure », voir entre autres Sahlins (1985), Alban Bensa (1996 et 2002), Ballard (2003). 
de vue structural comme selon une perspective diachronique du fait même de son processus historique de formation. Comme bien souvent en Océanie, on a emprunté une forme institutionnelle en en réadaptant le contenu aux habitus et aux contextes conjoncturels locaux. La mise en perspective ethnohistorique fait entrevoir également un référent culturel, une symbolique et des valeurs propres, une dimension à laquelle nous n'avons pu donner toute l'importance qu'il conviendrait dans cette déjà longue présentation, qui font du royaume de 'Uvea, non plus une « annexe territoriale » ou au mieux une variante $\mathrm{du}$ système monarchique tongien, mais une formation sociale originale inscrite dans son histoire, même si l'on doit encore s'interroger sur les raisons qui poussent la tradition orale de 'Uvea à toujours souligner et valoriser avec autant de constance son ancrage tongien.

\section{BIBLIOGRAPHIE}

Allen Melinda et Gertrude Bell, 1988. Lapita flaked stone assemblages. Sourcing, technological, and functional studies, in P. V. Kirch and T. L. Hunt (eds), Archaeology on the Lapita Cultural Complex: a critical Review, Seattle, The Burke Museum, pp. 83-98.

ANDERSON Atholl, 1991. The chronology of colonisation in New Zealand, Antiquity 65, pp. 767-95.

ANDERSON Atholl, Helen LEACH, Ian SMITH et Richard WALTER, 1994. Reconstitution of the Marquesan sequence in East Polynesian prehistory, with particular reference to Hane (MUH1), Archaeology in Oceania 29, pp. 29-52.

ANGLeviel Frédéric, 1994. Les missions à Wallis-etFutuna au XIX ${ }^{e}$ siècle, Bordeaux, CRET.

BALlard Chris, 2003. La fabrique de l'histoire. Événement, mémoire et récit dans les hautes terres de Nouvelle-Guinée, in I. Merle et M. Naepels (éds), Les rivages du temps. Histoire et anthropologie du Pacifique, Paris, L'Harmattan.

BALOuEt Jean-Christophe et Starrs L. Olson, 1987. A new extinct species of giant pigeon (Columbidae ducula) from archeological deposits of Wallis (Uvea) island, South Pacific, Processing of the Biological Society of Washington 100 (4), pp. 769-775.

BARRAU Jacques, 1963. L'agriculture des îles Wallis-etFutuna, Journal de la Société des Océanistes 19, pp. 157-171.

Bataillon Pierre, 1932. Langue d'Uvea (Wallis), Paris, P. Geuthner.

BEDFORD Stuart, 2003. The timing and nature of Lapita colonisation in Vanuatu: the haze begins to clear, in C. Sand (ed.), Pacific Archeology: Assessments and Prospects, Nouméa, service des Musées et du Patrimoine, pp. 147-158.
BENSA Alban, 1996. De la micro-histoire vers une anthropologie critique, in J. Revel (éd.), Jeux d'échelles, la micro-histoire à l'expérience, Paris, EHESS- Gallimard-Le Seuil, pp. 7-70.

—, 2000. Le chef kanak. Les modèles et l'histoire, in A. Bensa et I. Leblic (éds), En pays kanak, Paris, Éditions de la maison des sciences de l'homme, pp. $10-48$.

—, 2002. Les sciences sociales face à l'événement, Terrain 38, pp. 5-20.

Best Simon. 1984. Lakeba: The Prehistory of a Fijian Island, unpublished Ph. D. thesis Dissertation, University of Auckland, New Zealand,

BonNemAison Joël, 1996. Gens de pirogue et gens de la terre. Les fondements géographiques d'une identité : archipel du Vanuatu, Paris, ORSTOM.

BoTT Elisabeth (with the assistance of TAVI), 1982. Tongan Society at the Time of capitaine Cook's visits: Discussions with her Majesty Queen Salote Tupou, Wellington, The Polynesian Society, Memoir 44.

Brisse Henri et Michel Hoff, 1990. Contribution à l'étude des groupements végétaux des îles Wallis-etFutuna, Documents phytosociologiques n.s., XII, Camerino.

Burley David et Jeffrey ClARK, 2003. The archeology of Fiji/Western Polynesia in the post-Lapita era, in C. Sand (ed.), Pacific Archeology: Assessments and Prospects, Nouméa, service des Musées et du Patrimoine, pp. 235-254.

Burrows Edwin G., 1937. Ethnography of Uvea (Wallis Island), Honolulu, Bernice P. Bishop Museum, Bernice P. Bishop Museum Bulletin 145.

-, 1938a. Western Polynesia: A Study in Cultural Differentiation, Etnologiska Studier 7, Göteborg.

- 1938 b. Topography and culture on two Polynesian islands, Geographical Review 28, pp. 214-223.

_, 1939. Breed and border in Polynesia, American Anthropologist 41, pp. 1-21.

- 1940. Culture areas in Polynesia, Journal of the Polynesian Society 49, pp. 349-363.

-, 1945. Songs of Uvea and Futuna, Honolulu, Bernice P. Bishop Museum, Bernice P. Bishop Museum Bulletin 183.

Condominas Georges, 1976. Essai sur l'évolution des systèmes politiques thaïs, Etnos 41, pp. 7-67.

DAVIDSON Janet et Brian FAGAN, 1974. Excavations at site 12: Leuluasi, in R. C. Green and J. M. Davidson (eds), Archaeology in Western Samoa II, Auckland, Auckland Institute and Museum, Auckland Institute and Museum Bulletin 7, pp. 72-89.

Di Piazza Anne, 1988. Côté jardin. Ethnoarchéologie du paysage de Wallis-et-Futuna, Paris, ORSTOM, Études et thèses.

DouaIre-Marsaudon Françoise, 1998. Les premiers fruits. Parenté, identité sexuelle et pouvoirs en Polynésie occidentale (Tonga, Wallis-et-Futuna), Paris, 
CNRs Éditions - Éditions de la maison des sciences de l'homme.

Dumont Louis, 1957. For a Sociology of India, Contribution of Indian Sociology 1.

Dunis Serge, 2004. Le grand océan, le temps et l'espace du Pacifique, Genève, Paris, Georg, Ethnos.

Elbert Samuel et Torben Monberg, 1965. From the Two Canoes: Oral Traditions of Rennell and Bellona Islands, Honolulu, University of Hawaii Press.

FirTH Raymond, 1961. History and Traditions in Tikopia, Wellington, The Polynesian Society.

FrimigaCCI Daniel, 1980. Localisation écogéographique et utilisation de l'espace de quelques sites Lapita de Nouvelle-Calédonie : essai d'interprétation, Journal de la Société des Océanistes 66-67, pp. 5-11

—, 1990. Aux Temps de la Terre Noire, Ethnoarchéologie des îles Futuna et Alofi, Paris, Éditions Peeters, SELAF 321, Langues et cultures du Pacifique.

—, 1997. Puhi, the mythical paramount chief of Uvea and ancient links between Uvea and Tonga, in R. Blench et M. Spriggs (eds), Archaeology and Language I. Theorical and Methodological Orientations, Routledge, London, One World Archaeology 27, pp. 331-344.

—, 2000. La préhistoire de 'Uvea (Wallis). Chronologie et périodisation, Journal de la Société des Océanistes 111, pp. 135-163.

Frimigacci Daniel et Maurice Hardy, 1997. Des Archéologues, des Conquérants et des Forts (Talietumu, résidence tongienne d'Uvea), Versailles, Art Lys - Association socioculturelle pour la culture et l'art wallisiens et futuniens.

Frimigacci Daniel, Muni Keletaona, Claire Moyse-FAurie et Bernard VIENNE, 1995. Ko le fonu tu'a limulimua. La tortue au dos moussu. Textes de tradition orale de Futuna, Paris, Peeters.

Frimigacci Daniel et Siolesio Pilioko, n.d. Talanoa ki 'Uvea nei (Histoires d'Uvea), Textes de tradition orale, traduits en français, annotés et présentés par Daniel Frimigacci et Siolesio Pilioko, texte bilingue wallisien-français, ms. $150 \mathrm{p}$.

FrimigacCI Daniel, Jean-Pierre SioRat et Bernard VIENNE, 1982. Inventaire des sites archéologiques et ethnohistoriques de l'île d'Uvea, centre ORSTOM de Nouméa.

_, 1984. Inventaire et fouille des sites archéologiques et ethnohistoriques de l'île d'Uvea, centre orstom de Nouméa.

—, 1995. Un poisson nommé Uvea : Éléments d'ethnohistoire de Wallis, Nouméa, CTRDP.

Frimigacci Daniel et Bernard Vienne, 1987. How they become Polynesian: An ethnoarchaeological investigation of Futuna and Uvea, Man and Culture in Oceania special issue 3, pp. 117-119.

_, 2001. Wallis et Futuna : 3000 ans d'histoire, Nouméa, AJWFNC.
GaLIPAUd Jean-Christophe, 2000. The Lapita site of Atanoasao, Malo, Vanuatu, World Archeological Bulletin 12, pp. 41-55.

GIFFORD Edward W., 1924. Tongan myths and tales, Honolulu, Bernice P. Bishop Museum, Bernice P. Bishop Museum Bulletin 8.

—, 1929. Tongan Society, Honolulu, Bernice P. Bishop Museum, Bernice P. Bishop Museum Bulletin 61.

Graves Michael et David Addison, 1995. The Polynesian settlement of the Hawaiian archipelago. Integrating models and methods of archaeological interpretation, Word Archaeology 26, pp. 280-99.

Green Roger C., 1969a. Portable artefacts, in R. C. Green and J. Davidson (eds), Archaeology in Western Samoa 1, Auckland, Auckland Institute Museum, Auckland Institute and Museum Bulletin 6, pp. 166-175.

- (ed), 1969b. Archeology in Western Samoa, Auckland, Auckland Institute and Museum Bulletin 7-8.

_, 1976. Lapita Sites in the Santa Cruz Group, in R. C. Green et M. M. Cresswell (eds), Southeast Solomon Islands Cultural History: a Preliminary Survey, Wellington, Royal Society of New Zealand Bulletin 11, pp. 245-265.

Green Roger C. et Patrick V. KIRCH, 1997. Lapita exchange systems and their PolynesianTransformations: seeking explanatory models, in $\mathrm{M}$. Weisler (ed.), Prehistoric Long-Distance Interaction in Oceania: an interdisciplinary approach, Auckland, New Zealand Archeological Association Monograph 21, pp. 19-37.

Golson Jack, 1961. Report on New Zealand, Western Polynesia, New Caledonia and Fiji, Asian Perspectives 5, pp. 166-180.

Guiart Jean, 1952. Les origines de la population d'Ouvéa, Études mélanésiennes 6, pp. 26-35.

—, 1953. Mythes et chants polynésiens d'Ouvéa (Îles Loyalty), Journal of Polynesian Society 63 (2), pp. 126.

—, 1963. Structure de la chefferie en Mélanésie du Sud, Paris, Institut d'Ethnologie, Travaux et Mémoires de l'Institut d'Ethnologie LXVI.

—, 2005. Compte rendu de S. Dunis, 2004 (Le grand océan, le temps et l'espace du Pacifique, Genève, Paris, Georg, Ethnos), Bulletin des études océaniennes 302, pp. 175-193.

GuIot Hélène, 1997. Forêt taboue et représentation de l'environnement à 'Uvea (Wallis). Approche ethnoarchéologique, Journal de la Société des Océanistes 107, pp. 179-198.

—, 2000. 'Uvea-Wallis. Une île pêchée par les dieux, Musée des beaux-arts de Chartres.

HEDRICK John, n.d. Archeological Investigation of Malo Prehistory. Lapita Settlement Strategy in the Northern new Hebrides, Unpublished Ph. D. thesis, University of Pensylvania.

_, 1971. Lapita ZStyle Pottery from Malo Island, Journal of Polynesian Society 80 (1), pp. 2-15. 
Henquel Joseph, 1910. Talanoa ki 'Uvea nei, Lano, Wallis, Presses de la Mission.

Henry Fred, 1979. History of Samoa, Apia, Commercial Printers Limited.

Herda Phyllis, 1988. The Transformation of Traditional Tongan Policy : a Genealogical Consideration of the Past, Ph. D. Thesis, Canberra, ANU.

Hogbin Ian, 1931. A note on Rennell Island, Oceania 2, pp. 174-178.

Hooper Anthony et Judith Huntsman (eds), 1985. Transformations of Polynesian Culture, Auckland, The Polynesian Society, Polynesian Society Memoir 45.

Howard Alan et Robert Borofsky (eds), 1989. Developments in Polynesian Ethnology, Honolulu, University of Hawaii Press.

Huntsman Judith, 2004. Tokelau 1852 Exodus. A Story and its History, The Journal of Pacific History 39 (1), pp. 23-42.

Huntsman Judith et Anthony Hooper, 1991. Matagi Tokelau: History and Traditions of Tokelau, Translated from Takelauan and edited by J. Huntsman and A. Hooper, Apia and Suva, Office of Tokelau Affairs \& Institute of Pacific Studies.

-, 1996. Tokelau. A Historical Ethnography, Auckland, Auckland University Press.

JANETSKY Joel, 1980. Shell, bone, coral and urchin spine artefacts, in J. D. Jennings and R. N. Holmer (eds), Archaeological excavations in Western Samoa, Honolulu, Bishop Museum Press, Pacific Archaeologhical Records 32, pp. 123-31.

KIRCH Patrick V., 1976a. Rapport préliminaire sur les recherches archéologiques effectuées aux îles Wallis-et-Futuna (Polynésie occidentale), Journal de la Société des Océanistes 53, pp. 307-310.

- 1976b. Ethno-archaeological investigations in Futuna and Uvea (Western Polynesia): A preliminary report, Journal of the Polynesian Society 85-1, pp. 27-69.

-, 1978. Indigenous agriculture on Uvea (Western Polynesia), Economic Botany 32, pp. 157-181.

_, 1981. Lapitoid settlements on Futuna and Alofi, Western Polynesia, Archaeology in Oceania 17, p. $127-143$.

—, 1984. The Evolution of the Polynesian Chiefdoms, Cambridge, Cambridge University Press.

—, 1988. Niuatoputapu: The Prehistory of a Polynesian Chiefdom, Seattle, Burke Museum, T.H.Gomas Burke Memorial Washington State Museum Monograph 5.

-, 1990. Specialization and exchange in the Lapita Complex of Oceania (1600-500 BC), Asian Perspectives 29 (2), pp. 117-133.

-, 1993. Non ceramic portable artefacts from the To'oga site, in P. V. Kirch and T. L. Hunt (eds), The To'oga Site: Three millennia of Polynesian Occupation in the Manu'a Islands, American Samoa, Berkeley, University of California Press, Contributions of the University of California Archaeological Research Facility 51, pp 156-66.

-, 1994. The Wet and the Dry, Irrigation and Agricultural Intensification in Polynesia, Chicago and London, The University of Chicago Press.

- 1997. The Lapita Peoples. Ancestors of the Oceanic World, Cambridge, Massachusetts, Blackwell.

—, 2001. Hawaiki, Ancestral Polynesia. An Essay in Historical Anthropology, Cambridge, Cambridge University Press.

KIRCH Patrick V. et Paul Rosendahl, 1976. Early Anutan Settlement and the Position of Anuta in the Prehistory of the Southwest Pacific, in R. C. Green and M. M. Cresswell (eds), Southeast Solomon Islands Cultural History: a Preliminary Survey, Wellington, Royal Society of New Zealand Bulletin 11, pp. 225-244.

KIRCH Patrick V. et D. E. Yen, 1982. Tikopia. The Prehistory and Ecology of a Polynesian Outlier, Honolulu, Bernice P. Bishop Bulletin 238.

KIRCHHOFF Paul, 1955. The principles of clanship in human society, Journal of Anthropology 1, pp. 1-10.

LEACH Edmond, 1954. Political Systems of Highland Burma: a Study of Kachin Social Structure, London, The London School of Economics and Political Science.

LeleivaI Hapakuke Pierre, 2002. Contribution à la discussion relative au Talanoa ki 'Uvea nei du Révérend Père Joseph Henquel, Les cahiers de Wallis-etFutuna 2, pp. 15-29.

_, 2003. History and Archeology in 'Uvea (Wallis Island), Western Polynesia: Among Tradition, Myth and Science, in C. Sand (ed.), Pacific Archeology: Assessments and Prospects, Nouméa, service des Musées et du Patrimoine, pp. 337-342.

Lemoine Jacques, 1997. Féodalité Taï chez les Lü des Sipsong panna et les Taï blancs, noirs et rouges du Nord-Ouest du Vietnam. Péninsule 35, pp. 171-219.

MacGregGor Gordon, 1937. Ethnology of Tokelau Islands, Honolulu, Bernice P. Bishop Museum, Bernice P. Bishop Museum Bulletin 146.

—, 1943. The Gods of Rennell Island, in C. S. Coon and J. M. Andrew (eds), Studies in the Anthropology of Oceania and Asia, Cambridge, Harvard University, Papers of the Peabody Museum of American Archeology and Ethnology 20, pp. 32-37.

_, m.s., Fieldnotes, Rotuma, Bernice P. Bishop Museum.

Mahina 'Okusitino, 1992. The Tongan Traditional History, Tala E Fonua: A vernacular. Ecologycentred Historico-cultural Concept, M.A. Thesis Canberra, Australian National University.

MCKERn William, 1929. Archaeology of Tonga, Honolulu, Hawaii, Bernice P. Bishop Museum, Bulletin 60, Bayard Dominick expedition, Publication 13.

Mckoy P. C. and P. C. Gleghorn, 1988. Archeological excavations on Santa Cruz (Nendo), Southeast Solomon Islands: Summary Report, Archeology in Oceania 23 (3), pp. 104-114. 
Malau Atoloto, 1999. Horticulture, in Antonio Takasi, Atoloto Malau, Frédéric Angleviel et al., 101 mots pour comprendre Wallis-et-Futuna, Nouméa, Édition Île de Lumière, p. 109.

_, 2004, Culture et agriculture, in Filihau Asi Talatini et al., Uvea, Elise Huffer et Mikaele Tui (eds), translation from French to English by Neil Carruthers, Suva, Fiji/Mata'utu, Wallis, Institute of Pacific Studies University of the South Pacific/ Service des Affaires Culturelles de Wallis, 198 p.

MAYER Raymond, 1976. Les transformations de la tradition narrative à l'île Wallis (Uvea), Paris, Publications de la Société des Océanistes 38.

MEAd Margaret, 1930. Social Organization of Manu'a, Honolulu, Hawaii, Bernice P. Bishop Museum, Bulletin 60, Bayard Dominick expedition, Publication 76.

MeleiseA Malama, 1987. Lagaga: a Short History of Western Samoa, Suva, University of the South Pacific.

MonBerg Torben, 1991. Bellona Island Beliefs and Rituals, Honolulu, University of Hawaii Press.

OzANNE-Rivierre Françoise, 1994. Iaai Loanwords and Phonemic Changes in Fagauvea, in T. Dutton and D.T. Tryon (eds), Languages Contact and Change in the Austronesian World, Berlin and New York, Mouton de Gruyter, pp. 523-549.

PAwley Andrew et Roger GreEn, 1973. Dating the dispersal of the Oceanic languages, Oceanic Linguistics 12, pp. 1-67.

Pawley Andrew et Malcom Ross, 1993. Austronesian historical linguistics and culture history, Annual Review of Anthropology 22, pp. 425-459.

PInEDa Rufino et Jean-Christophe GaliPaud, 1998. Évidences archéologiques d'une surrection différentielle de l'île de Malo (Archipel du Vanuatu) au cours de l'holocène récent, Comptes rendus de l'Académie des sciences 327, pp. 777-779.

PONCET Alexandre, 1951. Cérémonial traditionnel de l'intronisation du roi de l'île d'Ouvéa (île Wallis) en Polynésie, Journal de la Société des Océanistes 7, pp. 237-242.

_, 1972. Histoire de l'île Wallis. Le Protectorat français, Paris, Publications de la Société des Océanistes 23.

RENCH Karl (ed.), 1981. Tohi fono o uvea, code de Wallis 1870, Canberra, Archipelago Press.

Rutherford Noel (ed.), 1977. A History of Tonga, Oxford, Oxford University Press.

SAHLins Marshall, 1958. Social Stratification in Polynesia, Seattle, American Ethnological Society.

—, 1968. Tribesmen, New Jersey, Prentice Hall.

_, 1981a. The stranger king, Journal of Pacific History 16 , pp. 107-132.

—, 1981b. Historical Metaphors and Mythical Realities: Structure in the Early History of the Sandwich Islands Kingdom, Ann Arbor, University of Michigan Press.
—, 1985. Islands of History, Chicago, University of Chicago Press.

SAND Christophe, 1993. A Preliminary Study of the Impact of the Tongan Maritime Chiefdom on the late Prehistoric Society of Uvea, Western Polynesia, in $\mathrm{M}$. et $\mathrm{R}$. Graves, manque référence complète, pp. $\mathrm{x}-\mathrm{x}$.

-, 1998. Uvéa : la préhistoire de Wallis, île de la Polynésie occidentale, Nouméa, Grain de Sable.

—, 1999. Empires maritimes préhistoriques dans le Pacifique : Ga'asialili et la mise en place d'une colonie tongienne à 'Uvea (Wallis, Polynésie occidentale), Journal de la Société des Océanistes 108 , pp. 103-124.

Smith Anita, 2002. An archaeology of West Polynesian Prehistory, Pandanus Book, Research School of Pacific and Asian Studies, The Australian National University, Canberra.

SprigGs Matthew, 1989. The dating of the Island Southeast Asia Neolithic. An attempt at chronometric hygiene and linguistic correlation, Antiquity 63, pp. 587-613.

—, 1990. Dating Lapita: Another view, in M. Spriggs (ed.), Lapita Design, Form and Composition, Canberra, The Australian National University Research School of Pacific Studies, Department of Prehistory, Occasional Papers in Prehistory 20, pp. 6-27.

_, 1997. The Island Melanesians, Cambridge Mass, Blackwell Publishers.

SprigGs Matthew and Atholl AnDERson, 1993 Late colonisation of East Polynesia, Antiquity 67, pp. 200-17.

STAIR John B., 1894. Samoa whence peopled? Journal of Polynesian Society 4, pp. 47-58.

Stuiver M., P. J. Reimer, J. W. Beck, G. S. Burr, K. A. Hughen, B. Kromer, F.G. v.d. Mccormac, J. Plicht and M. Spurk, 1998. References for datasets, Radiocarbon 40, pp. 1041-1083.

VALERI Valerio, 1985. Kingship and Sacrifice. Ritual and Society in Ancient Hawai'i, Chicago, The University of Chicago Press.

VIENNE Bernard, 1998. Réflexions sur quelques dimensions structurales de l'identité Océanienne, in D. Tryon et P. De Deckker (éds), Identités en mutation dans le Pacifique à l'aube du troisième millénaire, Bordeaux, CRET, Îles et archipels 26, pp. $71-$ 84.

—, 1999. Systèmes fonciers de Wallis-et-Futuna, in A. Leplaideur, Wallis-et-Futuna: Une transition agricole... à réguler?, Montpellier, CIRAD, miméo, pp. 44-53.

VIENNE Bernard et Yves Goudineau, 2001. L'État et les minorités ethniques. La place des populations montagnardes (chao khao) dans l'espace national, in S. Dovert (éd.), Thaïlande contemporaine, Paris, L'Harmattan - IRASEC, pp. 143-174. 
WARD Graham, 1979. Prehistoric Settlement and Economy in a Tropical Small Island Environnement: the Banks Islands, Insular Melanesia, unpublished $\mathrm{Ph}$. D. thesis, Canberra, ANU.
Williamson Robert W., 1924. The Social and Political Systems of Central Polynesia, Cambridge, Cambridge University Press. 